\title{
ON THE VARIATIONAL APPROXIMATION OF COMBINED SECOND AND FOURTH ORDER GEOMETRIC EVOLUTION EQUATIONS*
}

\author{
JOHN W. BARRETT ${ }^{\dagger}$, HARALD GARCKE $^{\ddagger}$, AND ROBERT NÜRNBERG ${ }^{\dagger}$
}

\begin{abstract}
We present a variational formulation of combined motion by minus the Laplacian of curvature and mean curvature flow, as well as related flows. The proposed scheme covers both the closed curve case and the case of curves that are connected via triple or quadruple junction points or intersect the external boundary. On introducing a parametric finite element approximation, we prove stability bounds and compare our scheme with existing approaches. The presented scheme has very good properties with respect to the equidistribution of mesh points and, if applicable, area conservation.
\end{abstract}

Key words. surface diffusion, (inverse) mean curvature flow, surface attachment limited kinetics, nonlinear curve evolution, triple junctions, parametric finite elements, Schur complement, tangential movement

AMS subject classifications. 65M60, 65M12, 35K55, 53C44

DOI. $10.1137 / 060653974$

1. Introduction. The motion of curves or surfaces driven by second or fourth order geometric evolution equations arises in many applications in materials science and, of course, in differential geometry. Well known is the mean curvature flow, where a hypersurface moves in the direction of its mean curvature vector. Also frequently arising are evolution laws where the normal speed of an evolving hypersurface is given as a function of mean curvature, in applications, such as image processing or mathematical physics. In mathematical physics the inverse mean curvature flow plays a role in the context of the positive mass conjecture. In materials science the motion of networks of curves or surfaces is also often important. The evolution of grain networks - arising in polycrystalline materials - is also given by mean curvature flow, where at junctions angle conditions have to hold.

Fourth order geometric evolution equations also frequently are found in geometry and materials science. The situation that the normal velocity of a hypersurface is given by minus the Laplacian of mean curvature is called surface diffusion. This evolution law arises in situations where the diffusion of material is restricted to interfacial regions; see, e.g., [36]. When many phases appear, networks also have to be taken into account. As the evolution law is of fourth order, additional conditions, which act as boundary conditions for the evolution law on the curves or surfaces, have to hold.

The goal of this paper is to propose and analyze a new approach to numerically solving geometric evolution laws of second and fourth order. The numerical method is variational and very flexible. In particular, it is possible to couple fourth and second order laws on the surfaces or at triple junctions in a straightforward way.

* Received by the editors March 9, 2006; accepted for publication (in revised form) October 25, 2006; published electronically May 10, 2007.

http://www.siam.org/journals/sisc/29-3/65397.html

$\dagger$ Department of Mathematics, Imperial College, London, SW7 2AZ, UK (j.barrett@imperial.ac.uk, robert.nurnberg@imperial.ac.uk).

${ }^{\ddagger}$ Naturwissenschaftliche Fakultät I - Mathematik, Universität Regensburg, 93040 Regensburg, Germany (harald.garcke@mathematik.uni-regensburg.de). 
There exist numerous methods for numerically solving geometric evolution equations. Approaches are based on, e.g., the parametric formulation, the graph formulation, the level set method, or the phase field approach. We do not intend to give an outline of all these methods, but rather refer to [14] for a comprehensive recent review. Our approach makes use of a fundamental idea in [16], which used the identity

$$
\Delta_{s} \vec{x}=\vec{\varkappa},
$$

where $\Delta_{s}$ is the surface Laplacian, $\vec{x}$ is the position vector, and $\vec{\varkappa}$ is the mean curvature vector, for the first time in order to design a finite element method for geometric partial differential equations and mean curvature flow; see also [17]. A second major idea stems from the paper [3], which came up with a splitting method and employed a Schur complement approach, in order to compute solutions of the surface diffusion law

$$
\mathcal{V}=-\Delta_{s} \varkappa,
$$

where $\varkappa$ is the sum of the principal curvatures of $\Gamma$ and $\mathcal{V}$ is the normal velocity of the surface.

The approaches of Dziuk and of Bänsch, Morin, and Nochetto both require that the parameterization evolve only in the normal direction. In the recent paper [6], the present authors introduced a novel parametric finite element approximation for surface diffusion that also allowed for a tangential movement of mesh points, which of course does not change the geometry. This new approach has the advantage that the discretization has very good properties with respect to the equidistribution of mesh points. In the case of surface diffusion of curves, a semidiscrete version of this scheme, i.e., a scheme where one discretizes only with respect to space, leads to a precise equidistribution of mesh points. With the new ansatz it is also possible to formulate the rather complicated conditions that need to hold at triple junction points in a natural variational way. In this paper we want to demonstrate that the ideas developed in [6] can also be used to compute other geometric evolution laws of both second and fourth order. For instance, the inverse mean curvature flow and an evolution flow which couples mean curvature flow to surface diffusion at triple junctions (see, e.g., [12]) can be approximated in the spirit of the approach proposed in $[6]$.

In this paper, we will consider evolution laws that couple fourth and second order geometric evolution laws. The coupling can be either through triple junctions or through a coupling that combines mean curvature flow and the fourth order surface diffusion flow via an appropriate interpolation; see [42] for applications of this flow in materials science. Let us introduce first the case of pure second order geometric evolution equations; that means in our context situations where the normal velocity is given by a function of mean curvature. For a closed hypersurface $\Gamma$ in $\mathbb{R}^{d}$, mean curvature flow is given by

$$
\mathcal{V}=\varkappa
$$

or, more generally, we are also going to consider flows of the form

$$
\mathcal{V}=f(\varkappa)
$$

where $f:(a, b) \rightarrow \mathbb{R}$ with $-\infty \leq a<b \leq \infty$ is a strictly monotonically increasing continuous function, e.g.,

$$
f(r):=|r|^{\beta-1} r, \quad \beta \in \mathbb{R}_{>0}
$$


see [35] and the references therein. For example, the evolution law (1.4), with (1.5) for $\beta=\frac{1}{3}$, has been studied in $[1,40,2]$.

We will also consider computations for (1.4) with

$$
f(r):=-r^{-1},
$$

i.e., the inverse mean curvature flow; see, e.g., $[29,32]$ for the origins of this flow in mathematical physics, and [31], and the references therein, for a consideration of this flow in differential geometry. To our knowledge, the only numerical results in the literature for (1.4) with (1.6) can be found in [38, 22], where a finite volume and a finite element approximation, respectively, of a regularized level set formulation of (1.4) with (1.6) are employed. We know of no direct approach for the approximation of the inverse mean curvature flow in the literature.

For a parameterization $\vec{x}(\rho, t) \in \mathbb{R}^{d}$ of $\Gamma,(1.4)$ can be written as a second order equation:

$$
\mathcal{V}:=\vec{x}_{t} \cdot \vec{\nu}=f(\varkappa), \quad \varkappa \vec{\nu}=\Delta_{s} \vec{x},
$$

where $\vec{\nu}$ is a unit normal to $\Gamma$. Note that because the tangential component $\vec{x}_{t}-$ $\left(\vec{x}_{t} \cdot \vec{\nu}\right) \vec{\nu}$ of the velocity $\vec{x}_{t}$ is not prescribed in (1.7), there exists a whole family of solutions $\vec{x}$, even though the evolution of $\Gamma$ is uniquely determined by (1.3). Our numerical scheme will directly discretize (1.7), in contrast to the scheme considered by Dziuk (see $[16,17]$ ), which discretizes $\vec{x}_{t}=\Delta_{s} \vec{x}$ and hence, on noting (1.1) and that $\vec{\varkappa}=\varkappa \vec{\nu}$, enforces a movement of the parameterization $\vec{x}$ in the normal direction only.

A version of (1.4) that preserves the enclosed volume is given by

$$
\mathcal{V}=f(\varkappa)-\frac{\int_{\Gamma} f(\varkappa) \mathrm{d} s}{\int_{\Gamma} 1 \mathrm{~d} s}
$$

the so-called conserved mean curvature flow, also called surface attachment limited kinetics (SALK), if $f(r):=r$. An intermediate law between (1.4), with $f(r):=r$, and (1.2) is the following evolution law:

$$
\mathcal{V}=-\Delta_{s}\left(\frac{1}{\alpha}-\frac{1}{\xi} \Delta_{s}\right)^{-1} \varkappa,
$$

where $\alpha, \xi \in \mathbb{R}_{>0}$. The flow (1.9) interpolates between surfaces diffusion (1.2) and SALK, (1.8) with $f(r):=r$, and was first discussed in [42]; see also [20]. It is similar to (1.2) and (1.8) in that the enclosed volume is conserved while the area of the hypersurface decreases. We observe that for $\alpha \rightarrow \infty$ and $\xi=1$ the solutions to (1.9) converge to solutions of (1.8) with $f(r):=r$, as has been shown in [21]. In the limit $\xi \rightarrow \infty$ and $\alpha=1$ the solutions to (1.9) should converge to solutions of the law (1.2), although to our knowledge no rigorous proof exists. Given a parameterization $\vec{x}(\rho, t) \in \mathbb{R}^{d}$ of $\Gamma,(1.9)$ can be written as a system of second order equations:

$$
\vec{x}_{t} \cdot \vec{\nu}=-\Delta_{s} y, \quad\left(\frac{1}{\alpha}-\frac{1}{\xi} \Delta_{s}\right) y=\varkappa, \quad \varkappa \vec{\nu}=\Delta_{s} \vec{x} .
$$

Analogously surface diffusion can be rewritten as

$$
\vec{x}_{t} \cdot \vec{\nu}=-\Delta_{s} \varkappa, \quad \varkappa \vec{\nu}=\Delta_{s} \vec{x} .
$$




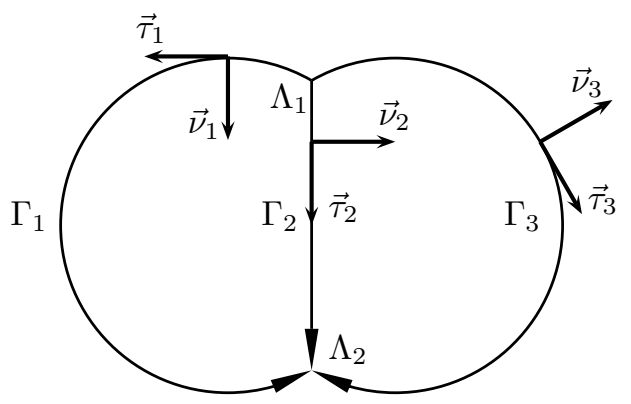

Fig. 1. The setup of $\Gamma=\left(\Gamma_{1}, \Gamma_{2}, \Gamma_{3}\right)$.

In section 2, we will consider a finite element approximation of a variational formulation of (1.11), as well as (1.7), (1.8), and (1.10), where throughout we will restrict our attention to the case $d=2$, i.e., curves in the plane. In addition, we will compare our approximation of (1.3) with two other parametric approximations in the literature, namely, $[17,13]$.

A network of curves under motion by mean curvature with triple junctions can also be considered. In the example network in Figure 1, let $\Gamma_{1}, \Gamma_{2}, \Gamma_{3}$ be the given curves in $\mathbb{R}^{d}, d=2$, that intersect at two triple junction points $\Lambda_{1}$ and $\Lambda_{2}$. Then the normal velocity for each curve is given by

$$
\mathcal{V}_{i}=\sigma_{i} \varkappa_{i}, \quad i=1 \rightarrow 3,
$$

where $\varkappa_{i}$ is the curvature of $\Gamma_{i}$ and $\sigma_{i}$ is the surface energy density of $\Gamma_{i}$. Let $\vec{\tau}_{i} \in \mathbb{R}^{d}$ be the unit tangent to $\Gamma_{i}$ pointing away from the triple junction point $\Lambda_{1}$ and towards point $\Lambda_{2}$. The curvature is said to be positive if $\Gamma_{i}$ is curved in the direction of the normal $\vec{\nu}_{i} \in \mathbb{R}^{d}$, which is the unique unit vector such that $\left(\vec{\tau}_{i}, \vec{\nu}_{i}\right)$ forms a positively orientated orthonormal system. For parameterizations $\vec{x}_{i}:[0,1] \times[0, T] \rightarrow \mathbb{R}^{d}$ of $\Gamma_{i}$, $i=1 \rightarrow 3,(1.12)$ can be written as a system of second order equations:

$$
\left(\vec{x}_{i}\right)_{t} \cdot \vec{\nu}_{i}=\sigma_{i} \varkappa_{i}, \quad \varkappa_{i} \vec{\nu}_{i}=\Delta_{s} \vec{x}_{i} .
$$

Then, in addition to (1.12), the following conditions have to hold at the triple junction points $\Lambda_{1}$ and $\Lambda_{2}$ :

the triple junction does not pull apart,

$$
\sigma_{1} \vec{\tau}_{1}+\sigma_{2} \vec{\tau}_{2}+\sigma_{3} \vec{\tau}_{3}=\overrightarrow{0}
$$

The condition (1.14a) is an attachment condition, and (1.14b) is Young's law, which is a balance-of-force equation at the triple junction. Young's law is equivalent to the angle condition $\frac{\sin \theta_{1}}{\sigma_{1}}=\frac{\sin \theta_{2}}{\sigma_{2}}=\frac{\sin \theta_{3}}{\sigma_{3}}$, where

$$
\theta_{1}=\varangle\left(\vec{\tau}_{2}, \vec{\tau}_{3}\right), \quad \theta_{2}=\varangle\left(\vec{\tau}_{3}, \vec{\tau}_{1}\right), \quad \text { and } \quad \theta_{3}=\varangle\left(\vec{\tau}_{1}, \vec{\tau}_{2}\right) .
$$

A variational formulation of $(1.13)$ with $(1.14 a, b)$ will form the basis for the scheme that we present in section 2 .

In most physical applications only triple junctions are of interest. However, in certain situations quadruple junctions are also possible. In the case that four interfaces meet at a quadruple junction the balance-of-force condition of the quadruple junction is

$$
\sigma_{1} \vec{\tau}_{1}+\sigma_{2} \vec{\tau}_{2}+\sigma_{3} \vec{\tau}_{3}+\sigma_{4} \vec{\tau}_{4}=\overrightarrow{0}
$$




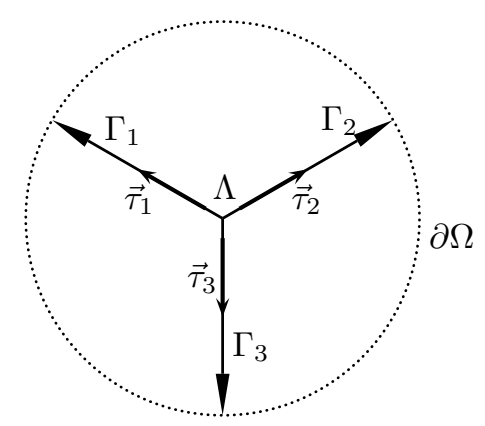

FIG. 2. The second possible setup of $\Gamma=\left(\Gamma_{1}, \Gamma_{2}, \Gamma_{3}\right)$.

where we use notation analogous to that in (1.14b); e.g., the four curves $\Gamma_{i}, i=1 \rightarrow 4$, meeting at the quadruple junction are parameterized such that the tangent vectors point away from the junction. In this case the angles at the quadruple junction are not specified, and in fact there is one degree of freedom. For details we refer to [10, 11, 24].

In [6] the possibility of curve intersections with a fixed external boundary $\partial \Omega$, where $\Omega$ is a domain in $\mathbb{R}^{d}$, was not considered (see Figure 2 for an example). To state the necessary conditions that have to hold at an intersection with the external boundary we assume that $\partial \Omega$ is given by a function $F \in C^{1}\left(\mathbb{R}^{d}\right)$ such that

$$
\partial \Omega=\left\{\vec{z} \in \mathbb{R}^{d}: F(\vec{z})=0\right\} \quad \text { and } \quad|\nabla F(\vec{z})|=1 \quad \forall \vec{z} \in \partial \Omega .
$$

Then, for three given curves $\Gamma_{i}, i=1 \rightarrow 3$, evolving according to (1.12), the conditions (1.14a,b) need to hold only at the triple junction $\Lambda=\vec{x}_{i}(0), i=1 \rightarrow 3$, while at the boundary intersection points $\vec{x}_{i}(1), i=1 \rightarrow 3$, the following conditions have to hold:

the curve endpoint remains attached to $\partial \Omega$,

$$
\vec{\tau}_{i} \cdot\left[\nabla F\left(\vec{x}_{i}(1)\right)\right]^{\perp}=0,
$$

where ${ }^{\perp}$ denotes the clockwise rotation by $\frac{\pi}{2}$. The second condition, $(1.17 \mathrm{~b})$, requires that forces at the outer boundary act only normal to the boundary, which is equivalent to a $90^{\circ}$ angle condition. This is the case when the two phases that meet the external boundary have the same contact energy with the boundary. If this is not the case, then condition (1.17b) has to be replaced by the angle condition

$$
\vec{\tau}_{i} \cdot \vec{t}=\vec{\tau}_{i} \cdot\left[\nabla F\left(\vec{x}_{i}(1)\right)\right]^{\perp}=\cos \alpha_{i},
$$

where $\vec{t}$ is a unit tangent to $\partial \Omega$. Condition (1.18) states that the curve $\Gamma_{i}$ intersects the outer boundary with a given angle $\alpha_{i}$; see, e.g., [23] for the physical background.

Before coupling fourth and second order geometric evolution equations at triple junctions, we now turn our attention to a network evolving according to surface diffusion. Using the same notation as above, we rewrite the evolution laws

$$
\mathcal{V}_{i}=-\sigma_{i} \Delta_{s} \varkappa_{i}, \quad i=1 \rightarrow 3,
$$

as

$$
\left(\vec{x}_{i}\right)_{t} \cdot \vec{\nu}_{i}=-\sigma_{i} \Delta_{s} \varkappa_{i}, \quad \varkappa_{i} \vec{\nu}_{i}=\Delta_{s} \vec{x}_{i} .
$$


These equations are coupled at triple junctions and have to fulfill boundary conditions at points that intersect the external boundary. At the triple junction we require in addition to $(1.14 \mathrm{a}, \mathrm{b})$ the conditions

$$
\begin{aligned}
& \sigma_{1} \varkappa_{1}+\sigma_{2} \varkappa_{2}+\sigma_{3} \varkappa_{3}=0, \\
& \sigma_{1} \vec{\tau}_{1} \cdot \nabla_{s} \varkappa_{1}=\sigma_{2} \vec{\tau}_{2} \cdot \nabla_{s} \varkappa_{2}=\sigma_{3} \vec{\tau}_{3} \cdot \nabla_{s} \varkappa_{3},
\end{aligned}
$$

where $\left.\nabla_{s}\right|_{\Gamma_{i}} \equiv \vec{\tau}_{i} \frac{\partial}{\partial s}$ with $s$ being the arclength. Equation (1.21a) follows from the continuity of chemical potentials, and (1.21b) is a flux balance condition; see [28] for details. At an intersection with an external boundary we require $(1.17 \mathrm{a}, \mathrm{b})$ and the following no-flux condition at the external boundary:

$$
\nabla_{s} \varkappa_{i}=\overrightarrow{0}
$$

Finally, another possible setup is to require motion by mean curvature only on a subset of the given curves, while the remaining curves move by surface diffusion. This is of relevance, e.g., in thermal grooving [36], in interface motion in polycrystalline two-phase materials [10], and in the evolution of boundaries in the electromigration of intergranular voids [7]. For parameterizations $\vec{x}_{i}:[0,1] \times[0, T] \rightarrow \mathbb{R}^{d}$ of $\Gamma_{i}$, $i=1 \rightarrow K_{C}$, this gives rise to the following system of equations:

$$
\begin{aligned}
\left(\vec{x}_{i}\right)_{t} \cdot \vec{\nu}_{i} & =\sigma_{i} \varkappa_{i}, \quad i=1 \rightarrow i_{0}, \quad\left(\vec{x}_{i}\right)_{t} \cdot \vec{\nu}_{i}=-\sigma_{i} \Delta_{s} \varkappa_{i}, \quad i=i_{0}+1 \rightarrow K_{C}, \\
\varkappa_{i} \vec{\nu}_{i} & =\Delta_{s} \vec{x}_{i}, \quad i=1 \rightarrow K_{C},
\end{aligned}
$$

subject to the triple junction conditions $(1.14 \mathrm{a}, \mathrm{b})$. In addition, we require that at triple junctions

$$
\sigma_{i} \vec{\tau}_{i} \cdot \nabla_{s} \varkappa_{i}=\sigma_{j} \vec{\tau}_{j} \cdot \nabla_{s} \varkappa_{j}
$$

for all interfaces $i, j \in\left\{i_{0}+1, \ldots, K_{C}\right\}$ which are present at the junction. Further, we require that

$$
\sum_{i \in T_{S D}} \sigma_{i} \varkappa_{i}=0
$$

where $T_{S D} \subset\left\{i_{0}+1, \ldots, K_{C}\right\}$ are the interfaces present at the triple junction which move according to surface diffusion. We note that for practical applications of (1.23) only triple junctions, where one curve that moves by mean curvature flow and two curves moving by surface diffusion meet, are of interest. A variational formulation of (1.23) with the appropriate conditions at triple junctions and along the fixed external boundary will also be considered in section 2 .

As for previous work on the approximation of curve networks, we refer to $[9,43$, 37]. Also relevant is [8], where (1.12) and (1.14a,b) are derived from an Allen-Cahn system. A parametric finite element approximation of the mean curvature flow of a curve that intersects an external boundary was considered in [15]. A level set approach for mean curvature flow of curve networks has been considered in [34, 44, 41]. A phase field model for the combined motion of mean curvature flow and surface diffusion, as well as SALK, was considered in [7]. A phase field model for a mean curvature flow system is considered in [27], and a surface diffusion flow system in [4].

This paper is organized as follows. In section 2 we formulate a finite element approximation of problem (1.13) and derive stability bounds. Here we first introduce our 
approximation for the simpler case of a closed curve, (1.7), and then generalize that scheme to cover (1.8), as well as (1.20) and $(1.14 \mathrm{a}, \mathrm{b})$ in the case of a triple junction configuration, as in Figure 1. We indicate how to generalize the approach to a configuration as in Figure 2, as well as to an arbitrary setup of curves, triple junctions, and external boundary intersections. We will also indicate how to combine this approach with the algorithm presented in [6] to yield a scheme for combined mean curvature flow and surface diffusion. Finally, we consider the adaptation of our scheme to approximate (1.10). In section 3 we present a large number of numerical computations and compare our results, where possible, with those from other parametric algorithms in the literature.

\section{Finite element approximation.}

2.1. Closed curves. We introduce the following finite element approximation. Let $J:=\mathbb{R} / \mathbb{Z}=\bigcup_{j=1}^{N} J_{j}, N \geq 3$, be a decomposition of $J$ into intervals given by the nodes $q_{j}, J_{j}=\left[q_{j-1}, q_{j}\right]$. Let $h_{j}=\left|J_{j}\right|$ and $h=\max _{j=1 \rightarrow N} h_{j}$ be the maximal length of a grid element. Then the necessary finite element spaces are defined as follows:

$$
\underline{V}_{0}^{h}:=\left\{\vec{\chi} \in C\left(J, \mathbb{R}^{d}\right):\left.\vec{\chi}\right|_{J_{j}} \text { is linear } \forall j=1 \rightarrow N\right\}=:\left[W_{0}^{h}\right]^{d} \subset H^{1}\left(J, \mathbb{R}^{d}\right),
$$

where $W_{0}^{h} \subset H^{1}(J, \mathbb{R})$ is the space of scalar continuous (periodic) piecewise linear functions, with $\left\{\phi_{l}\right\}_{l=1}^{N}$ denoting the standard basis of $W_{0}^{h}$. Throughout this paper, we make use of the periodicity of $J$, i.e., $q_{N} \equiv q_{0}, q_{N+1} \equiv q_{1}$, and so on.

In addition, let $0=t_{0}<t_{1}<\cdots<t_{M-1}<t_{M}=T$ be a partitioning of $[0, T]$ into possibly variable time steps $\tau_{m}:=t_{m+1}-t_{m}, m=0 \rightarrow M-1$. We set $\tau:=\max _{m=0 \rightarrow M-1} \tau_{m}$. Let $\vec{X}^{m} \in \underline{V}_{0}^{h}$ be an approximation to $\vec{x}\left(\cdot, t_{m}\right)$, and similarly $\kappa^{m} \in W_{0}^{h}$ for $\varkappa\left(\cdot, t_{m}\right)$.

For scalar and vector functions $u, v \in L^{2}\left(J, \mathbb{R}^{(d)}\right)$ we introduce the $L^{2}$ inner product $\langle\cdot, \cdot\rangle_{m}$ over the current polygonal curve $\Gamma^{m}$, which is described by the vector function $\vec{X}^{m} \in \underline{V}_{0}^{h}$, as follows:

$$
\langle u, v\rangle_{m}:=\int_{\Gamma^{m}} u \cdot v \mathrm{~d} s=\int_{J} u \cdot v\left|\vec{X}_{\rho}^{m}\right| \mathrm{d} \rho,
$$

where, here and throughout, $\vec{X}_{\rho}^{m}$ denotes the derivative of $\vec{X}^{m}$ with respect to $\rho$, where $\rho \in[0,1]$ is the parameterization variable, and.$\left.^{*}\right)$ denotes an expression with or without the superscript $*$, and similarly for subscripts. In addition, if $u, v$ are piecewise continuous, with possible jumps at the nodes $\left\{q_{j}\right\}_{j=1}^{N}$, we define the mass lumped inner product $\langle\cdot, \cdot\rangle_{m}^{h}$ as

$$
\langle u, v\rangle_{m}^{h}:=\frac{1}{2} \sum_{j=1}^{N}\left|\vec{X}^{m}\left(q_{j}\right)-\vec{X}^{m}\left(q_{j-1}\right)\right|\left[(u \cdot v)\left(q_{j}^{-}\right)+(u \cdot v)\left(q_{j-1}^{+}\right)\right],
$$

where we define $u\left(q_{j}^{ \pm}\right):=\lim _{\varepsilon \backslash 0} u\left(q_{j} \pm \varepsilon\right)$. Furthermore, we note that $\nabla_{s} u . \nabla_{s} v=$ $\frac{u_{\rho} \cdot v_{\rho}}{\left|\vec{X}_{\rho}^{m}\right|^{2}}$ and $\vec{\nu}^{m}=-\frac{\left(\vec{X}_{\rho}^{m}\right)^{\perp}}{\left|\vec{X}_{\rho}^{m}\right|}$.

We propose the following approximation to (1.7): Find $\left\{\vec{X}^{m+1}, \kappa^{m+1}\right\} \in \underline{V}_{0}^{h} \times W_{0}^{h}$ such that

$$
\begin{aligned}
\left\langle\frac{\vec{X}^{m+1}-\vec{X}^{m}}{\tau_{m}}, \chi \vec{\nu}^{m}\right\rangle_{m}^{h}-\left\langle f\left(\kappa^{m+1}\right), \chi\right\rangle_{m}^{h}=0 & \forall \chi \in W_{0}^{h}, \\
\left\langle\kappa^{m+1} \vec{\nu}^{m}, \vec{\eta}\right\rangle_{m}^{h}+\left\langle\nabla_{s} \vec{X}^{m+1}, \nabla_{s} \vec{\eta}\right\rangle_{m}=0 & \forall \vec{\eta} \in \underline{V}_{0}^{h},
\end{aligned}
$$


where, as noted above, the inner products $\langle\cdot, \cdot\rangle_{m}^{(h)}$ as well as $\nabla_{s}$ depend on $m$.

In order to approximate (1.8), we adapt (2.3a) to

$$
\left\langle\frac{\vec{X}^{m+1}-\vec{X}^{m}}{\tau_{m}}, \chi \vec{\nu}^{m}\right\rangle_{m}^{h}-\left\langle f\left(\kappa^{m+1}\right), \chi\right\rangle_{m}^{h}=-\frac{\left\langle f\left(\kappa^{m}\right), 1\right\rangle_{m}^{h}}{\langle 1,1\rangle_{m}}\langle 1, \chi\rangle_{m} \quad \forall \chi \in W_{0}^{h},
$$

where $\kappa^{0} \in W_{0}^{h}$ is suitably chosen; see section 3 .

Remark 2.1. We note that in $(2.3 \mathrm{a}, \mathrm{b})$, and throughout this paper, we use piecewise linear finite elements, e.g., as defined in (2.1). This is a sensible choice for the type of evolution equations in consideration, both from a theoretical and practical points of view. It is also in line with other approaches in the literature; see, e.g., $[16,17,13,15,19,3]$. However, from a computational point of view, one could also choose to use higher order finite elements.

Before we can proceed to prove existence and uniqueness of (2.3a,b), we have to make the following very mild assumption.

and set

$\left(\mathcal{A}_{0}\right) \quad$ Let $\left|\vec{X}_{\rho}^{m}\right|>0$ for almost all $\rho \in J$. For $j=1 \rightarrow N$, let $\vec{\nu}_{j-\frac{1}{2}}^{m}:=-\left.\frac{\left(\vec{X}_{\rho}^{m}\right)^{\perp}}{\left|\vec{X}_{\rho}^{m}\right|}\right|_{J_{j}}$,

$$
\begin{aligned}
\vec{\omega}_{j}^{m} & :=\frac{\left|\vec{X}^{m}\left(q_{j}\right)-\vec{X}^{m}\left(q_{j-1}\right)\right| \vec{\nu}_{j-\frac{1}{2}}^{m}+\left|\vec{X}^{m}\left(q_{j+1}\right)-\vec{X}^{m}\left(q_{j}\right)\right| \vec{\nu}_{j+\frac{1}{2}}^{m}}{\left|\vec{X}^{m}\left(q_{j}\right)-\vec{X}^{m}\left(q_{j-1}\right)\right|+\left|\vec{X}^{m}\left(q_{j+1}\right)-\vec{X}^{m}\left(q_{j}\right)\right|} \\
& =\frac{-\left[\vec{X}^{m}\left(q_{j+1}\right)-\vec{X}^{m}\left(q_{j-1}\right)\right]^{\perp}}{\left|\vec{X}^{m}\left(q_{j}\right)-\vec{X}^{m}\left(q_{j-1}\right)\right|+\left|\vec{X}^{m}\left(q_{j+1}\right)-\vec{X}^{m}\left(q_{j}\right)\right|} .
\end{aligned}
$$

Then we further assume that $\operatorname{dim} \operatorname{span}\left\{\vec{\omega}_{j}^{m}\right\}_{j=1}^{N}=d=2$.

Remark 2.2. We note that one can interpret $\vec{\omega}_{j}^{m}$ as a weighted normal defined at the node $\vec{X}^{m}\left(q_{j}\right)$ of the curve $\Gamma^{m}$, where in general $\left|\vec{\omega}_{j}^{m}\right|<1$. In addition, we note that $\left(\mathcal{A}_{0}\right)$ is violated only in very rare occasions. For example, it always holds for curves without self intersections; see [6, Remark 2.2] for details.

THEOREM 2.3. Let the assumption $\left(\mathcal{A}_{0}\right)$ hold, and assume that $f:(a, b) \rightarrow \mathbb{R}$ with $-\infty \leq a<0<b \leq \infty$ is strictly increasing, continuous, and such that $f((a, b))=\mathbb{R}$. Then there exists a unique solution $\left\{\vec{X}^{m+1}, \kappa^{m+1}\right\} \in \underline{V}_{0}^{h} \times W_{0}^{h}$ to the system $(2.3 \mathrm{a}, \mathrm{b})$.

Proof. For later developments involving networks of curves, we first discuss the linear case when $f(r):=r$, and so existence follows from uniqueness. To investigate the latter, we consider the following system: Find $\{\vec{X}, \kappa\} \in \underline{V}_{0}^{h} \times W_{0}^{h}$ such that

$$
\begin{aligned}
\left\langle\vec{X}, \chi \vec{\nu}^{m}\right\rangle_{m}^{h}-\tau_{m}\langle\kappa, \chi\rangle_{m}^{h}=0 & \forall \chi \in W_{0}^{h}, \\
\left\langle\kappa \vec{\nu}^{m}, \vec{\eta}\right\rangle_{m}^{h}+\left\langle\nabla_{s} \vec{X}, \nabla_{s} \vec{\eta}\right\rangle_{m}=0 & \forall \vec{\eta} \in \underline{V}_{0}^{h} .
\end{aligned}
$$

Choosing $\chi=\kappa \in W_{0}^{h}$ in (2.6a) and $\vec{\eta}=\vec{X} \in \underline{V}_{0}^{h}$ in (2.6b) yields that

$$
\left\langle\nabla_{s} \vec{X}, \nabla_{s} \vec{X}\right\rangle_{m}+\tau_{m}\langle\kappa, \kappa\rangle_{m}^{h}=0
$$

It follows from (2.7) that $\kappa \equiv 0$ and $\vec{X} \equiv \vec{X}^{c} \in \mathbb{R}^{d}$, and hence that

$$
\left\langle\vec{X}^{c}, \chi \vec{\nu}^{m}\right\rangle_{m}^{h}=0 \quad \forall \chi \in W_{0}^{h} .
$$

Choosing $\chi=\phi_{j}$ in (2.8) yields that $\vec{X}^{c} \cdot \vec{\omega}_{j}^{m}=0$ for all $j=1 \rightarrow N$. It follows from assumption $\left(\mathcal{A}_{0}\right)$ that $\vec{X}^{c}=\overrightarrow{0}$. Hence we have shown that $(2.3 \mathrm{a}, \mathrm{b})$ with $f(r):=r$ has a unique solution $\left\{\vec{X}^{m+1}, \kappa^{m+1}\right\} \in \underline{V}_{0}^{h} \times W_{0}^{h}$. 
For a general function $f:(a, b) \rightarrow \mathbb{R}$ fulfilling the assumptions of the theorem, we can rewrite (2.3a,b), on noting (2.2) and (2.5), as: Find $\vec{X}^{m+1} \in \underline{V}_{0}^{h}$ such that

$$
\left\langle\nabla_{s} \vec{X}^{m+1}, \nabla_{s} \vec{\eta}\right\rangle_{m}+\left\langle f^{-1}\left(\frac{\vec{X}^{m+1}-\vec{X}^{m}}{\tau_{m}} \cdot \vec{\omega}^{m}\right), \vec{\eta} \cdot \vec{\omega}^{m}\right\rangle_{m}^{h}=0 \quad \forall \vec{\eta} \in \underline{V}_{0}^{h},
$$

where $\vec{\omega}^{m}:=\sum_{j=1}^{N} \vec{\omega}_{j}^{m} \phi_{j}$. Then $\kappa^{m+1} \in W_{0}^{h}$ is uniquely determined from

$$
\kappa^{m+1}\left(q_{j}\right)=f^{-1}\left(\frac{\vec{X}^{m+1}\left(q_{j}\right)-\vec{X}^{m}\left(q_{j}\right)}{\tau_{m}} \cdot \vec{\omega}_{j}^{m}\right), \quad j=1 \rightarrow N .
$$

On noting our assumption $\left(\mathcal{A}_{0}\right)$, similarly as in the linear case above, it follows that (2.9) is the Euler-Lagrange variation of the strictly convex minimization problem

$$
\min _{\vec{\eta} \in \underline{V}_{0}^{h}}\left[\frac{1}{2}\left\langle\nabla_{s} \vec{\eta}, \nabla_{s} \vec{\eta}\right\rangle_{m}+\tau_{m}\left\langle\Phi\left(\frac{\vec{\eta}-\vec{X}^{m}}{\tau_{m}} \cdot \vec{\omega}^{m}\right), 1\right\rangle_{m}^{h}\right]
$$

where $\Phi$ is an antiderivative of $f^{-1}$. We note that $\Phi: \mathbb{R} \rightarrow \mathbb{R}$ is strictly convex with $\Phi^{\prime}(f(0))=f^{-1}(f(0))=0$, and hence we obtain that $\Phi$ is bounded from below and coercive. Therefore there exists a unique solution $\vec{X}^{m+1} \in \underline{V}_{0}^{h}$ to (2.9), and hence a unique solution $\left\{\vec{X}^{m+1}, \kappa^{m+1}\right\} \in \underline{V}_{0}^{h} \times W_{0}^{h}$ to $(2.3 \mathrm{a}, \mathrm{b})$.

The above proof immediately applies to the case when (2.3a) is replaced by (2.4). We remark also that we still obtain uniqueness for strictly increasing continuous functions $f:(a, b) \rightarrow \mathbb{R}$ with $-\infty \leq a<b \leq \infty$. This follows, since $\Phi$ defined as above is still strictly convex. Existence cannot be established as easily as above, because $\Phi$ is not coercive any longer. This discussion is relevant, e.g., for $f(r)=-r^{-1}$ with $r \in(0, \infty)$, which is the case of the inverse mean curvature flow if $\varkappa(\cdot, 0)>0$. In this case we obtain that $\Phi:(-\infty, 0) \rightarrow \mathbb{R}$ is defined as $\Phi(r)=\ln |r|$.

In addition, stability results for $(2.3 \mathrm{a}, \mathrm{b})$ and the variants involving (2.4) can be established in certain cases; see Theorem 2.10, and the ensuing comment, below. For example, the term $\left\langle\kappa^{m+1}, \kappa^{m+1}\right\rangle_{m}^{h}$ in the analogue of (2.38) is replaced by $\left\langle f\left(\kappa^{m+1}\right), \kappa^{m+1}\right\rangle_{m}^{h}$, which once again is nonnegative if $f$ is monotonically increasing with $f(0)=0$. Of course, it may be computationally more convenient to consider a linearized version of (2.3a). For example, for (1.5) with $\beta \geq 1$ one could replace (2.3a) by

$$
\left\langle\frac{\vec{X}^{m+1}-\vec{X}^{m}}{\tau_{m}}, \chi \vec{\nu}^{m}\right\rangle_{m}^{h}-\left\langle\frac{f\left(\kappa^{m}\right)}{\kappa^{m}} \kappa^{m+1}, \chi\right\rangle_{m}^{h}=0 \quad \forall \chi \in W_{0}^{h},
$$

with $\kappa^{0} \in W_{0}^{h}$ suitably chosen. Once again, it is then straightforward to prove existence and uniqueness and to derive a stability result for this scheme.

In order to solve the (nonlinear) algebraic systems arising from $(2.3 \mathrm{a}, \mathrm{b})$ and its generalizations, we apply a Schur complement approach. For later developments involving networks of curves, we describe it here for the linear case when $f(r):=r$. However, it easily caries over to nonlinear $f$; see $\left(2.14 \mathrm{a}\right.$,b) below. Let $\overrightarrow{I d}_{n} \in\left(\mathbb{R}^{d \times d}\right)^{n \times n}$ be the identity matrix, and similarly for $I d_{n} \in \mathbb{R}^{n \times n}$. We introduce also the matrices $\vec{N}_{0} \in\left(\mathbb{R}^{d}\right)^{N \times N}, M_{0} \in \mathbb{R}^{N \times N}$, and $\vec{A}_{0} \in\left(\mathbb{R}^{d \times d}\right)^{N \times N}$ with entries

$$
\left[M_{0}\right]_{k l}:=\left\langle\phi_{k}, \phi_{l}\right\rangle_{m}^{h}, \quad\left[\vec{N}_{0}\right]_{k l}:=\int_{\Gamma^{m}} \pi^{h}\left[\phi_{k} \phi_{l}\right] \vec{\nu}^{m} \mathrm{~d} s, \quad\left[\vec{A}_{0}\right]_{k l}:=\left\langle\nabla_{s} \phi_{k}, \nabla_{s} \phi_{l}\right\rangle_{m} \overrightarrow{I d}_{1},
$$


where $\pi^{h}: C(J, \mathbb{R}) \rightarrow W_{0}^{h}$ is the standard interpolation operator at the nodes $\left\{q_{j}\right\}_{j=1}^{N}$. We can then formulate $(2.3 \mathrm{a}, \mathrm{b})$ with $f(r):=r$ as: Find $\left\{\delta \vec{X}^{m+1}, \kappa^{m+1}\right\} \in\left(\mathbb{R}^{d}\right)^{N} \times \mathbb{R}^{N}$ such that

$$
\left(\begin{array}{cc}
\tau_{m} M_{0} & -\vec{N}_{0}^{T} \\
\vec{N}_{0} & \vec{A}_{0}
\end{array}\right)\left(\begin{array}{c}
\kappa^{m+1} \\
\delta \vec{X}^{m+1}
\end{array}\right)=\left(\begin{array}{c}
0 \\
-\vec{A}_{0} \vec{X}^{m}
\end{array}\right)
$$

where, with an obvious abuse of notation, $\delta \vec{X}^{m+1}=\left(\delta \vec{X}_{1}^{m+1}, \ldots, \delta \vec{X}_{N}^{m+1}\right)^{T}$ and $\kappa^{m+1}=\left(\kappa_{1}^{m+1}, \ldots, \kappa_{N}^{m+1}\right)^{T}$ are the vectors of coefficients with respect to the standard basis for $\vec{X}^{m+1}-\vec{X}^{m}$ and $\kappa^{m+1}$, respectively. We can transform (2.12) into

$$
\begin{aligned}
\kappa^{m+1} & =\frac{1}{\tau_{m}} M_{0}^{-1} \vec{N}_{0}^{T} \delta \vec{X}^{m+1}, \\
\left(\vec{A}_{0}+\frac{1}{\tau_{m}} \vec{N}_{0} M_{0}^{-1} \vec{N}_{0}^{T}\right) \delta \vec{X}^{m+1} & =-\vec{A}_{0} \vec{X}^{m} .
\end{aligned}
$$

As (2.13b) is clearly symmetric and positive definite under our assumption $\left(\mathcal{A}_{0}\right)$, there exists a unique solution to (2.13b). Moreover, the solution to $(2.13 \mathrm{a}, \mathrm{b})$ uniquely solves (2.3a,b) with $f(r):=r$.

For later purposes, we note that for the approximation $(2.3 \mathrm{a}, \mathrm{b})$ with nonlinear $f$, the linear Schur system $(2.13 \mathrm{a}, \mathrm{b})$ has to be replaced by the corresponding nonlinear system

$$
\begin{aligned}
\kappa^{m+1} & =f^{-1}\left[\frac{1}{\tau_{m}} M_{0}^{-1} \vec{N}_{0}^{T} \delta \vec{X}^{m+1}\right], \\
\vec{A}_{0} \delta \vec{X}^{m+1}+\vec{N}_{0} f^{-1}\left[\frac{1}{\tau_{m}} M_{0}^{-1} \vec{N}_{0}^{T} \delta \vec{X}^{m+1}\right] & =-\vec{A}_{0} \vec{X}^{m}
\end{aligned}
$$

where $f^{-1}(z) \in \mathbb{R}^{N}$ is defined by $\left[f^{-1}(z)\right]_{i}:=f^{-1}\left(z_{i}\right), i=1 \rightarrow N$, for any $z \in \mathbb{R}^{N}$.

Remark 2.4. In section 3, we will report on computations for our scheme (2.3a,b) and compare our results in the case of $f(r):=r$ with two other schemes in the literature. The first scheme is from [17] and can be formulated as: Find $\vec{X}^{m+1} \in \underline{V}_{0}^{h}$ such that

$$
\left\langle\frac{\vec{X}^{m+1}-\vec{X}^{m}}{\tau_{m}}, \vec{\eta}\right\rangle_{m}^{h}+\left\langle\nabla_{s} \vec{X}^{m+1}, \nabla_{s} \vec{\eta}\right\rangle_{m}=0 \quad \forall \vec{\eta} \in \underline{V}_{0}^{h} .
$$

The system (2.15) is a discretization of the variational formulation of

$$
\vec{x}_{t}=\vec{\varkappa}, \quad \vec{\varkappa}:=\varkappa \vec{\nu}=\Delta_{s} \vec{x},
$$

as opposed to (1.7) with $f(r):=r$. From (2.10), we see that our scheme (2.3a,b) with $f(r):=r$ can be rewritten as: Find $\vec{X}^{m+1} \in \underline{V}_{0}^{h}$ such that

$$
\left\langle\frac{\vec{X}^{m+1}-\vec{X}^{m}}{\tau_{m}} \cdot \vec{\omega}^{m}, \vec{\eta} \cdot \vec{\omega}^{m}\right\rangle_{m}^{h}+\left\langle\nabla_{s} \vec{X}^{m+1}, \nabla_{s} \vec{\eta}\right\rangle_{m}=0 \quad \forall \vec{\eta} \in \underline{V}_{0}^{h},
$$

which clearly highlights the key difference between the two schemes. The second scheme is from [13] and can be formulated as follows: Find $\vec{X}^{m+1} \in \underline{V}_{0}^{h}$ such that

$$
\int_{J}\left|\vec{X}_{\rho}^{m}\right|^{2} \pi^{h}\left[\frac{\vec{X}^{m+1}-\vec{X}^{m}}{\tau_{m}} \cdot \vec{\eta}\right] \mathrm{d} \rho+\int_{J} \vec{X}_{\rho}^{m+1} \cdot \vec{\eta}_{\rho} \mathrm{d} \rho=0 \quad \forall \vec{\eta} \in \underline{V}_{0}^{h},
$$


or equivalently

$$
\left\langle\left|\vec{X}_{\rho}^{m}\right| \frac{\vec{X}^{m+1}-\vec{X}^{m}}{\tau_{m}}, \vec{\eta}\right\rangle_{m}^{h}+\left\langle\left|\vec{X}_{\rho}^{m}\right| \nabla_{s} \vec{X}^{m+1}, \nabla_{s} \vec{\eta}\right\rangle_{m}=0 \quad \forall \vec{\eta} \in \underline{V}_{0}^{h} .
$$

We note that the scheme (2.15) changes the approximation of $\vec{x}$ predominantly in the normal direction (recall $(2.16)$ ), whereas the scheme $(2.3 \mathrm{a}, \mathrm{b})$ proposed in this paper, as well as (2.18), also induce tangential changes. This is a crucial difference. Without movement in the tangential direction, clustering and coalescence of nodes can occur, which leads to a breakdown of the algorithm. In addition, we note that the schemes (2.15) and (2.18) do not easily generalize to the case of nonlinear $f$, in contrast to our scheme $(2.3 \mathrm{a}, \mathrm{b})$. However, it should be noted that the numerical analysis of the schemes (2.15) and (2.18) is well developed, in that error bounds in the semidiscrete case have been derived; see $[17,13]$. This is something that we hope to achieve for our scheme in the future.

Remark 2.5. Similarly to [6, Remark 2.3], one can consider a continuous-in-time semidiscrete version of our scheme $(2.3 \mathrm{a}, \mathrm{b})$. In particular, we let

$$
\begin{aligned}
\left\langle\vec{X}_{t}, \chi \vec{\nu}^{h}\right\rangle^{h}-\langle f(\kappa), \chi\rangle^{h}=0 & \forall \chi \in W_{0}^{h}, \\
\left\langle\kappa \vec{\nu}^{h}, \vec{\eta}\right\rangle^{h}+\left\langle\nabla_{s} \vec{X}, \nabla_{s} \vec{\eta}\right\rangle=0 & \forall \vec{\eta} \in \underline{V}_{0}^{h},
\end{aligned}
$$

where we always integrate over the current curve $\Gamma^{h}$, described by $\vec{X}$, and so $\vec{\nu}^{h}=$ $-\frac{\left(\vec{X}_{\rho}\right)^{\perp}}{\left|\vec{X}_{\rho}\right|}$ and $\langle\cdot, \cdot\rangle^{(h)}$ is the same as $\langle\cdot, \cdot\rangle_{m}^{(h)}$ with $\Gamma^{m}$ and $\vec{X}^{m}$ replaced by $\Gamma^{h}$ and $\vec{X}$, respectively. It is then possible to show that the scheme $(2.19 \mathrm{a}, \mathrm{b})$ will always equidistribute the nodes along $\Gamma^{h}$ if the corresponding intervals are not locally parallel; see [6, Remark 2.4] for details. Although it does not appear possible to prove an analogue for the fully discrete scheme $(2.3 \mathrm{a}, \mathrm{b})$, in practice we see that the nodes are moved tangentially so that they will eventually be equidistributed; see section 3 for details.

While the scheme (2.18) from [13] also induces a tangential movement of vertices, it does not appear possible to show an analogous result for that scheme.

2.1.1. Intermediate evolution laws. In this subsection we consider the intermediate motion (1.10). We introduce the following approximation to (1.10): Find $\left\{\vec{X}^{m+1}, Y^{m+1}, \kappa^{m+1}\right\} \in \underline{V}_{0}^{h} \times\left[W_{0}^{h}\right]^{2}$ such that

$$
\begin{aligned}
\left\langle\frac{\vec{X}^{m+1}-\vec{X}^{m}}{\tau_{m}}, \chi \vec{\nu}^{m}\right\rangle_{m}^{h}-\left\langle\nabla_{s} Y^{m+1}, \nabla_{s} \chi\right\rangle_{m}=0 & \forall \chi \in W_{0}^{h}, \\
\frac{1}{\xi}\left\langle\nabla_{s} Y^{m+1}, \nabla_{s} \chi\right\rangle_{m}+\frac{1}{\alpha}\left\langle Y^{m+1}, \chi\right\rangle_{m}^{h}-\left\langle\kappa^{m+1}, \chi\right\rangle_{m}^{h}=0 & \forall \chi \in W_{0}^{h}, \\
\left\langle\kappa^{m+1} \vec{\nu}^{m}, \vec{\eta}\right\rangle_{m}^{h}+\left\langle\nabla_{s} \vec{X}^{m+1}, \nabla_{s} \vec{\eta}\right\rangle_{m}=0 & \forall \vec{\eta} \in \underline{V}_{0}^{h} .
\end{aligned}
$$

Remark 2.6. The scheme $(2.20 \mathrm{a}-\mathrm{c})$ is close in concept to the approximation for (1.11) in [6]: Find $\left\{\vec{X}^{m+1}, \kappa^{m+1}\right\} \in \underline{V}_{0}^{h} \times W_{0}^{h}$ such that

$$
\begin{aligned}
\left\langle\frac{\vec{X}^{m+1}-\vec{X}^{m}}{\tau_{m}}, \chi \vec{\nu}^{m}\right\rangle_{m}^{h}-\left\langle\nabla_{s} \kappa^{m+1}, \nabla_{s} \chi\right\rangle_{m}=0 & \forall \chi \in W_{0}^{h}, \\
\left\langle\kappa^{m+1} \vec{\nu}^{m}, \vec{\eta}_{m}^{h}+\left\langle\nabla_{s} \vec{X}^{m+1}, \nabla_{s} \vec{\eta}\right\rangle_{m}=0\right. & \forall \vec{\eta} \in \underline{V}_{0}^{h} .
\end{aligned}
$$


A semidiscrete version of $(2.21 \mathrm{a}, \mathrm{b})$ enjoys exact area conservation and an equidistribution of vertices; see [6, Remarks 2.3 and 2.4] for the relevant details. We note that the same holds true for the semidiscrete analogue of $(2.20 \mathrm{a}-\mathrm{c})$.

THEOREM 2.7. Let assumption $\left(\mathcal{A}_{0}\right)$ hold. Then there exists a unique solution $\left\{\vec{X}^{m+1}, Y^{m+1}, \kappa^{m+1}\right\} \in \underline{V}_{0}^{h} \times\left[W_{0}^{h}\right]^{2}$ to the system (2.20a-c). Moreover, we have that

$$
\left|\Gamma^{k}\right|+\frac{1}{\alpha} \sum_{m=0}^{k-1} \tau_{m}\left|\nabla_{s} Y^{m+1}\right|_{m}^{2}+\xi \sum_{m=0}^{k-1} \tau_{m}\left|\kappa^{m+1}-\frac{1}{\alpha} Y^{m+1}\right|_{m, h}^{2} \leq\left|\Gamma^{0}\right|
$$

for all $k=1 \rightarrow M$, where $|\cdot|_{m(, h)}^{2}:=\langle\cdot, \cdot\rangle_{m}^{(h)}$.

Proof. The uniqueness proof is a straightforward adaption of the proof of Theorem 2.3. As $(2.20 \mathrm{a}-\mathrm{c})$ is linear, existence follows from uniqueness, and the latter is easily established for the relevant equations

$$
\begin{array}{rlrl}
\left\langle\vec{X}, \chi \vec{\nu}^{m}\right\rangle_{m}^{h}-\tau_{m}\left\langle\nabla_{s} Y, \nabla_{s} \chi\right\rangle_{m} & =0 & & \forall \chi \in W_{0}^{h}, \\
\frac{1}{\xi}\left\langle\nabla_{s} Y, \nabla_{s} \chi\right\rangle_{m}+\frac{1}{\alpha}\langle Y, \chi\rangle_{m}^{h}-\langle\kappa, \chi\rangle_{m}^{h}=0 & & \forall \chi \in W_{0}^{h}, \\
\left\langle\kappa \vec{\nu}^{m}, \vec{\eta}\right\rangle_{m}^{h}+\left\langle\nabla_{s} \vec{X}, \nabla_{s} \vec{\eta}\right\rangle_{m}=0 & \forall \vec{\eta} \in \underline{V}_{0}^{h},
\end{array}
$$

on choosing $\chi=\frac{\alpha}{\xi} \kappa, \chi=\tau_{m}(\alpha \kappa-Y)$, and $\vec{\eta}=\frac{\alpha}{\xi} \vec{X}$, respectively. Combining yields that

$$
\frac{\alpha}{\xi}\left\langle\nabla_{s} \vec{X}, \nabla_{s} \vec{X}\right\rangle_{m}+\frac{\tau_{m}}{\xi}\left\langle\nabla_{s} Y, \nabla_{s} Y\right\rangle_{m}+\frac{\tau_{m}}{\alpha}\langle\alpha \kappa-Y, \alpha \kappa-Y\rangle_{m}^{h}=0 .
$$

It follows from (2.23) that $\vec{X}=\vec{X}^{c} \in \mathbb{R}^{d}, Y=Y^{c} \in \mathbb{R}$, and $\kappa=\frac{Y^{c}}{\alpha} \in \mathbb{R}$, and hence, similarly to $(2.8)$ on recalling assumption $\left(\mathcal{A}_{0}\right)$, that $\vec{X}^{c}=\overrightarrow{0}$ and $Y^{c}=0$. Hence we have existence of a unique solution $\left\{\vec{X}^{m+1}, Y^{m+1}, \kappa^{m+1}\right\} \in \underline{V}_{0}^{h} \times\left[W_{0}^{h}\right]^{2}$ to the system $(2.20 \mathrm{a}-\mathrm{c})$. Finally, choosing $\chi=\frac{\alpha}{\xi} \kappa^{m+1}, \chi=\tau_{m}\left(\alpha \kappa^{m+1}-Y^{m+1}\right)$, and $\vec{\eta}=\frac{\alpha}{\xi}\left(\vec{X}^{m+1}-\vec{X}^{m}\right)$ in $(2.20 \mathrm{a}-\mathrm{c})$ gives, similarly to (2.23), that

$$
\left\langle\nabla_{s} \vec{X}^{m+1}, \nabla_{s}\left(\vec{X}^{m+1}-\vec{X}^{m}\right)\right\rangle_{m}+\frac{\tau_{m}}{\alpha}\left|\nabla_{s} Y^{m+1}\right|_{m}^{2}+\xi \tau_{m}\left|\kappa^{m+1}-\frac{1}{\alpha} Y^{m+1}\right|_{m, h}^{2}=0 .
$$

Combining (2.24) with the closed curve analogue of (2.40), below, yields (2.22).

On recalling the definitions (2.11) and on similarly introducing the matrix $A_{0} \in$ $\mathbb{R}^{N \times N}$, we can reformulate $(2.20 \mathrm{a}-\mathrm{c})$ as: Find $\left\{\delta \vec{X}^{m+1}, Y^{m+1} \kappa^{m+1}\right\} \in\left(\mathbb{R}^{d}\right)^{N} \times\left[\mathbb{R}^{N}\right]^{2}$ such that

$$
\left(\begin{array}{ccc}
0 & \tau_{m} A_{0} & -\vec{N}_{0}^{T} \\
-M_{0} & \frac{1}{\xi} A_{0}+\frac{1}{\alpha} M_{0} & 0 \\
\vec{N}_{0} & 0 & \vec{A}_{0}
\end{array}\right)\left(\begin{array}{c}
\kappa^{m+1} \\
Y^{m+1} \\
\delta \vec{X}^{m+1}
\end{array}\right)=\left(\begin{array}{c}
0 \\
0 \\
-\vec{A}_{0} \vec{X}^{m}
\end{array}\right),
$$

where, with obvious abuse of notation, $\delta \vec{X}^{m+1}, Y^{m+1}$, and $\kappa^{m+1}$ are the vectors of coefficients with respect to the standard basis of $\vec{X}^{m+1}-\vec{X}^{m}, Y^{m+1}$, and $\kappa^{m+1}$, respectively. Introducing the inverse $S_{0}$ of $A_{0}$ restricted on the set $\left(\text { ker } A_{0}\right)^{\perp} \equiv$ $(\operatorname{span}\{1\})^{\perp}$, where $1:=(1, \ldots, 1)^{T} \in \mathbb{R}^{N}$, and noting that the first equation in $(2.25)$ 
implies that $1^{T} \vec{N}_{0}^{T} \delta \vec{X}^{m+1}=0$, one can transform (2.25) to

$$
\begin{aligned}
Y^{m+1}= & \frac{1}{\tau_{m}} S_{0} \vec{N}_{0}^{T} \delta \vec{X}^{m+1}+\mu 1, \\
\kappa^{m+1}= & \frac{1}{\tau_{m}}\left(\frac{1}{\alpha} S_{0}+\frac{1}{\xi} M_{0}^{-1}\right) \vec{N}_{0}^{T} \delta \vec{X}^{m+1}+\frac{\mu}{\alpha} 1, \\
\left(\vec{A}_{0}+\right. & \left.\frac{1}{\tau_{m}} \vec{N}_{0}\left[\frac{1}{\alpha} S_{0}+\frac{1}{\xi} M_{0}^{-1}\right] \vec{N}_{0}^{T}\right) \delta \vec{X}^{m+1}=-\vec{A}_{0} \vec{X}^{m}-\frac{\mu}{\alpha} \vec{N}_{0} 1, \\
& \left(\delta \vec{X}^{m+1}\right)^{T} \vec{N}_{0} 1=0,
\end{aligned}
$$

where $\mu=\frac{1^{T} Y^{m+1}}{1^{T} 1} \in \mathbb{R}$ is unknown. We introduce also the orthogonal projection $\vec{\Pi}_{0}$ onto $\mathcal{R}_{0}^{\perp}:=\left\{\vec{X} \in\left(\mathbb{R}^{d}\right)^{N}: \vec{X}^{T} \vec{N}_{0} 1=0\right\}$ by $\vec{\Pi}_{0}:=\overrightarrow{I d}_{N}-\frac{\vec{w} \vec{w}^{T}}{\vec{w}^{T} \vec{w}}$, where $\vec{w}:=\vec{N}_{0} 1$. Then (2.26c), on noting that $\vec{\Pi}_{0} \delta \vec{X}^{m+1}=\delta \vec{X}^{m+1}$, is replaced by

$$
\vec{\Pi}_{0}\left(\vec{A}_{0}+\frac{1}{\tau_{m}} \vec{N}_{0}\left[\frac{1}{\alpha} S_{0}+\frac{1}{\xi} M_{0}^{-1}\right] \vec{N}_{0}^{T}\right) \vec{\Pi}_{0} \delta \vec{X}^{m+1}=-\vec{\Pi}_{0} \vec{A}_{0} \vec{X}^{m} .
$$

As $(2.20 \mathrm{a}-\mathrm{c})$ has a unique solution, it is easily established that there exists a unique solution to (2.27). Moreover, the system (2.27) is symmetric and positive definite on $\mathcal{R}_{0}^{\perp}$. For details of a similar situation involving surface diffusion and triple junctions, we refer to [6, Theorem 2.4].

2.2. Triple junctions. In this section, we consider the case where a network of curves meeting at triple junction points moves under motion by mean curvature. Here the curves can meet at triple junction points or can intersect the external boundary $\partial \Omega$. For ease of exposition, from now on we consider the two cases of three curves $\left(\Gamma_{1}, \Gamma_{2}, \Gamma_{3}\right)$ with surface energies $\sigma:=\left(\sigma_{1}, \sigma_{2}, \sigma_{3}\right)$ meeting either at two triple junction points $\Lambda_{1}$ and $\Lambda_{2}$, as in Figure 1, or meeting at a single triple junction point $\Lambda$ and each intersecting the external boundary $\partial \Omega$, as in Figure 2. In particular, we note the stated choices of the direction of the unit tangents. We will outline also how the ideas presented for these cases can be carried over to an arbitrary setup of curves; see Remark 2.13 below.

We begin with the first setup. The main idea for the necessary trial (三 test) spaces is to make sure that the conditions $(1.14 \mathrm{a}, \mathrm{b})$ hold either essentially or weakly at the triple junctions. Here we will enforce condition (1.14a) explicitly through the trial space, whereas condition (1.14b) will be enforced weakly, similarly to a Neumann boundary condition for a standard second order elliptic PDE.

Let $I:=[0,1]$ be the unit interval, and let $I=\bigcup_{j=1}^{N_{i}} I_{j}^{i}, i=1 \rightarrow 3$, be decompositions of $I$ into intervals $I_{j}^{i}=\left[q_{j-1}^{i}, q_{j}^{i}\right]$ based on the nodes $\left\{q_{j}^{i}\right\}_{j=0}^{N_{i}}, N_{i} \geq 2$. Let $h_{j}^{i}=\left|I_{j}^{i}\right|$ and $h=\max _{i=1 \rightarrow 3} \max _{j=1 \rightarrow N_{i}} h_{j}^{i}$ be the maximal length of a grid element. Let $\underline{V}:=\left\{\left(\vec{\chi}_{1}, \vec{\chi}_{2}, \vec{\chi}_{3}\right) \in\left[C\left(I, \mathbb{R}^{d}\right)\right]^{3}: \vec{\chi}_{1}=\vec{\chi}_{2}=\vec{\chi}_{3}\right.$ on $\left.\partial I\right\}$ and $W_{\mathcal{M}}:=$ $\left\{\left(\chi_{1}, \chi_{2}, \chi_{3}\right) \in[C(I, \mathbb{R})]^{3}\right\}$. The appropriate finite element spaces are then defined by

$$
\underline{V}^{h}:=\left\{\left(\vec{\chi}_{1}, \vec{\chi}_{2}, \vec{\chi}_{3}\right) \in \underline{V}:\left.\vec{\chi}_{i}\right|_{I_{j}^{i}} \text { is linear } \forall j=1 \rightarrow N_{i}, i=1 \rightarrow 3\right\} \subset \underline{V}
$$

and similarly for the space of scalar functions $W_{\mathcal{M}}^{h} \subset W_{\mathcal{M}}$.

Recall the time partitioning $\left\{\tau_{m}\right\}_{m=0}^{M-1}$, and let $\vec{X}^{m} \in \underline{V}^{h}$ be an approximation to $\vec{x}\left(\cdot, t_{m}\right) \equiv\left(\vec{x}_{1}, \vec{x}_{2}, \vec{x}_{3}\right)\left(\cdot, t_{m}\right)$, and similarly $\kappa^{m} \in W_{\mathcal{M}}^{h}$ for $\varkappa\left(\cdot, t_{m}\right)$. We introduce the $\sigma$ weighted $L^{2}$ inner product $\langle\cdot, \cdot\rangle_{m}$ and the mass lumped inner product $\langle\cdot, \cdot\rangle_{m}^{h}$ over 
the current surface $\Gamma^{m}:=\left(\Gamma_{1}^{m}, \Gamma_{2}^{m}, \Gamma_{3}^{m}\right)$, which is described by the vector function $\vec{X}^{m} \in \underline{V}^{h}$, for scalar and vector functions $u, v \in\left[L^{2}\left(I, \mathbb{R}^{(d)}\right)\right]^{3}$ as follows:

$$
\begin{aligned}
\langle u, v\rangle_{m} & :=\int_{\Gamma^{m}} u \cdot v \mathrm{~d} s:=\sum_{i=1}^{3} \sigma_{i} \int_{I} u_{i} \cdot v_{i}\left|\left(\vec{X}_{i}^{m}\right)_{\rho}\right| \mathrm{d} \rho \\
\langle u, v\rangle_{m}^{h} & :=\sum_{i=1}^{3} \frac{\sigma_{i}}{2} \sum_{j=1}^{N_{i}}\left|\vec{X}_{i}^{m}\left(q_{j}^{i}\right)-\vec{X}_{i}^{m}\left(q_{j-1}^{i}\right)\right|\left[\left(u_{i} \cdot v_{i}\right)\left(\left[q_{j}^{i}\right]^{-}\right)+\left(u_{i} \cdot v_{i}\right)\left(\left[q_{j-1}^{i}\right]^{+}\right)\right] .
\end{aligned}
$$

In addition, we note that

$$
\left.\left(\nabla_{s} u . \nabla_{s} v\right)\right|_{\Gamma_{i}^{m}}=\frac{\left(u_{i}\right)_{\rho} \cdot\left(v_{i}\right)_{\rho}}{\left|\left(\vec{X}_{i}^{m}\right)_{\rho}\right|^{2}},\left.\quad \vec{\nu}^{m}\right|_{\Gamma_{i}^{m}}=-\frac{\left(\vec{X}_{i}^{m}\right)_{\rho}^{\perp}}{\left|\left(\vec{X}_{i}^{m}\right)_{\rho}\right|}, \quad i=1 \rightarrow 3 .
$$

We then propose the following approximation to (1.13) with (1.14a,b): Find $\left\{\vec{X}^{m+1}\right.$, $\left.\kappa^{m+1}\right\} \in \underline{V}^{h} \times W_{\mathcal{M}}^{h}$ such that

$$
\begin{array}{cc}
\left\langle\frac{\vec{X}^{m+1}-\vec{X}^{m}}{\tau_{m}}, \chi \vec{\nu}^{m}\right\rangle_{m}^{h}-\left\langle\sigma \kappa^{m+1}, \chi\right\rangle_{m}^{h}=0 & \forall \chi \in W_{\mathcal{M}}^{h}, \\
\left\langle\kappa^{m+1} \vec{\nu}^{m}, \vec{\eta}\right\rangle_{m}^{h}+\left\langle\nabla_{s} \vec{X}^{m+1}, \nabla_{s} \vec{\eta}\right\rangle_{m}=0 & \forall \vec{\eta} \in \underline{V}^{h} .
\end{array}
$$

Observe that $(2.30 \mathrm{a}, \mathrm{b})$ was derived from (1.13) using integration by parts and the definition of the space $\underline{V}^{h}$. On noting that $\left.\left(\nabla_{s} \vec{X}^{m+1}\right)\right|_{\Gamma_{i}^{m}}$ approximates $\vec{\tau}_{i} \vec{\tau}_{i}^{T}, i=$ $1 \rightarrow 3$, we see that $(2.30 \mathrm{~b})$ weakly approximates Young's law (1.14b) at the triple junction points $\Lambda_{1}$ and $\Lambda_{2}$.

An extension of the scheme (2.18) that incorporates intersections with an external boundary was given in [15]. The authors considered a setup where a single curve intersects a fixed external boundary, and, for their fully discrete scheme, introduced the following trial and test space: For a given $\vec{X}^{m} \in C\left(I, \mathbb{R}^{d}\right)$,

$$
\underline{\widetilde{V}}\left(\vec{X}^{m}\right):=\left\{\vec{\chi} \in C\left(I, \mathbb{R}^{d}\right): \vec{\chi} \cdot \nabla F\left(\vec{X}^{m}\right)=0 \text { on } \partial I\right\},
$$

and in addition $\widetilde{V}^{h}\left(\vec{X}^{m}\right):=\left\{\vec{\chi} \in \tilde{V}\left(\vec{X}^{m}\right):\left.\vec{\chi}\right|_{I_{j}^{1}}\right.$ is linear $\left.\forall j=1 \rightarrow N_{1},\right\} \subset$ $\widetilde{V}\left(\vec{X}^{m}\right)$. Then their fully discrete approximation can be formulated as: Find $\delta \vec{X}^{m+1} \in$ $\widetilde{V}^{h}\left(\vec{X}^{m}\right)$, where $\vec{X}^{m+1}:=\vec{X}^{m}+\delta \vec{X}^{m+1}$ such that

$$
\int_{I}\left|\vec{X}_{\rho}^{m}\right|^{2} \pi^{h}\left[\frac{\delta \vec{X}^{m+1}}{\tau_{m}} \cdot \vec{\eta}\right] \mathrm{d} \rho+\int_{I} \vec{X}_{\rho}^{m+1} \cdot \vec{\eta}_{\rho} \mathrm{d} \rho=0 \quad \forall \vec{\eta} \in \underline{\widetilde{V}}^{h}\left(\vec{X}^{m}\right) .
$$

We now adapt the definition (2.31) to the setup as depicted in Figure 2. For a given $\vec{X}^{m} \in\left[C\left(I, \mathbb{R}^{d}\right)\right]^{3}$, let

$$
\begin{aligned}
& \underline{V}_{\partial}\left(\vec{X}^{m}\right):=\left\{\left(\vec{\chi}_{1}, \vec{\chi}_{2}, \vec{\chi}_{3}\right) \in\left[C\left(I, \mathbb{R}^{d}\right)\right]^{3}:\right. \vec{\chi}_{1}(0)=\vec{\chi}_{2}(0)=\vec{\chi}_{3}(0) \\
& \text { and } \\
&\left.\vec{\chi}_{i}(1) . \nabla F\left(\vec{X}_{i}^{m}(1)\right)=0 \quad \forall i=1 \rightarrow 3\right\} .
\end{aligned}
$$

The finite element space $\underline{V}_{\partial}^{h}\left(\vec{X}^{m}\right)$ is then defined accordingly, similarly to (2.28). Moreover, the system $(2.30 \mathrm{a}, \mathrm{b})$ is then adapted to: Find $\left\{\delta \vec{X}^{m+1}, \kappa^{m+1}\right\} \in \underline{V}_{\partial}^{h}\left(\vec{X}^{m}\right) \times$ 
$W_{\mathcal{M}}^{h}$, where $\vec{X}^{m+1}:=\vec{X}^{m}+\delta \vec{X}^{m+1}$, such that

$$
\begin{array}{ll}
\left\langle\frac{\delta \vec{X}^{m+1}}{\tau_{m}}, \chi \vec{\nu}^{m}\right\rangle_{m}^{h}-\left\langle\sigma \kappa^{m+1}, \chi\right\rangle_{m}^{h}=0 & \forall \chi \in W_{\mathcal{M}}^{h}, \\
\left\langle\kappa^{m+1} \vec{\nu}^{m}, \vec{\eta}\right\rangle_{m}^{h}+\left\langle\nabla_{s} \vec{X}^{m+1}, \nabla_{s} \vec{\eta}\right\rangle_{m}=0 & \forall \vec{\eta} \in \underline{V}_{\partial}^{h}\left(\vec{X}^{m}\right) .
\end{array}
$$

Once again $(2.33 \mathrm{a}, \mathrm{b})$ was derived from (1.13) using integration by parts and the definition of the space $\underline{V}_{\partial}^{h}\left(\vec{X}^{m}\right)$. On recalling that $\left.\left(\nabla_{s} \vec{X}^{m+1}\right)\right|_{\Gamma_{i}^{m}}$ approximates $\vec{\tau}_{i} \vec{\tau}_{i}^{T}$, $i=1 \rightarrow 3$, we see that (2.33b) weakly approximates Young's law (1.14b) at the triple junction point $\Lambda$ and (1.17b) at the boundary intersections. In order to approximate the general contact angle condition (1.18), we need to replace (2.33b) by

$$
\begin{aligned}
& \left\langle\kappa^{m+1} \vec{\nu}^{m}, \vec{\eta}\right\rangle_{m}^{h}+\left\langle\nabla_{s} \vec{X}^{m+1}, \nabla_{s} \vec{\eta}\right\rangle_{m} \\
& \quad=\sum_{i=1}^{3} \sigma_{i} \frac{\left[\nabla F\left(\vec{X}_{i}^{m}(1)\right)\right]^{\perp}}{\left|\nabla F\left(\vec{X}_{i}^{m}(1)\right)\right|} \cdot \vec{\eta}_{i}(1) \cos \alpha_{i} \quad \forall \vec{\eta} \in \underline{V}_{\partial}^{h}\left(\vec{X}^{m}\right) .
\end{aligned}
$$

Furthermore, the constraint $\delta \vec{X}^{m+1} \in \underline{V}_{\partial}^{h}\left(\vec{X}^{m}\right)$ weakly enforces (1.17a), as it is a linearized approximation of these constraints. In particular, for curved boundaries the equations $F\left(\vec{X}_{i}^{m+1}(1)\right)=0, i=1 \rightarrow 3$, are only approximately satisfied. Similarly to $[15$, p. 651$]$ it formally follows, on assuming that $F\left(\vec{X}_{i}^{0}(1)\right)=0$ for $i=1 \rightarrow 3$, that

$$
\left|F\left(\vec{X}_{i}^{m+1}(1)\right)\right| \leq \sum_{k=0}^{m}\left|F\left(\vec{X}_{i}^{k+1}(1)\right)-F\left(\vec{X}_{i}^{k}(1)\right)\right|=\mathcal{O}(\tau), \quad i=1 \rightarrow 3 .
$$

Hence, for small time steps the endpoints of the curve segments should stay close to $\partial \Omega$, and this is what one observes in practice; see section 3 for details. However, one could also employ a projection step that orthogonally projects $\vec{X}^{m+1}$ onto $\partial \Omega$ at every time step, which would have the advantage of satisfying (1.17a) exactly throughout the evolution. But this complicates the stability proof below; hence our preference for the approximation $(2.33 \mathrm{a}, \mathrm{b})$. However, we do include numerical results using both approaches in section 3 .

We remark that as the parameterization $\vec{X}^{m+1}$ does not "see" the boundary for $\rho<1$, it is possible that the evolving curves touch the external boundary at some interior point, in which case the evolution is no longer described by the approximation. We note that for a convex domain this cannot happen for mean curvature flow; see [39]. However, in the surface diffusion case this can happen even for convex domains.

Remark 2.8. We note that, as stated in [15, p. 640], it is not clear how to naturally generalize the scheme (2.32) to triple junctions, as there are severe difficulties with how to approximate the condition (1.14b) within that scheme.

Before we can proceed to proving existence and uniqueness for $(2.30 \mathrm{a}, \mathrm{b})$, we have to make the following very mild assumption, the analogue of $\left(\mathcal{A}_{0}\right)$ for a network of curves.

(A) Let $\left|\left(\vec{X}_{i}^{m}\right)_{\rho}\right|>0$ for almost all $\rho \in I, i=1 \rightarrow 3$. Let

$$
\vec{\nu}_{i, j-\frac{1}{2}}^{m}:=-\left.\frac{\left(\vec{X}_{i}^{m}\right)_{\rho}^{\perp}}{\left|\left(\vec{X}_{i}^{m}\right)_{\rho}\right|}\right|_{I_{j}^{i}}, j=1 \rightarrow N_{i},
$$


and set

$$
\begin{aligned}
\vec{\omega}_{i, j}^{m} & :=\frac{\left|\vec{X}_{i}^{m}\left(q_{j}^{i}\right)-\vec{X}_{i}^{m}\left(q_{j-1}^{i}\right)\right| \vec{\nu}_{i, j-\frac{1}{2}}^{m}+\left|\vec{X}_{i}^{m}\left(q_{j+1}^{i}\right)-\vec{X}_{i}^{m}\left(q_{j}^{i}\right)\right| \vec{\nu}_{i, j+\frac{1}{2}}^{m}}{\left|\vec{X}_{i}^{m}\left(q_{j}^{i}\right)-\vec{X}_{i}^{m}\left(q_{j-1}^{i}\right)\right|+\left|\vec{X}_{i}^{m}\left(q_{j+1}^{i}\right)-\vec{X}_{i}^{m}\left(q_{j}^{i}\right)\right|} \\
j & =1 \rightarrow N_{i}-1, i=1 \rightarrow 3 .
\end{aligned}
$$

Then we assume further that $\operatorname{dim} \operatorname{span}\left\{\left\{\vec{\omega}_{i, j}^{m}\right\}_{j=1}^{N_{i}-1}\right\}_{i=1}^{3}=d=2$.

The assumption $(\mathcal{A})$ basically assures that none of the curves $\Gamma_{i}^{m}, i=1 \rightarrow 3$, is a "zig zagging" connection between the two triple junctions points $\Lambda_{1}$ and $\Lambda_{2}$. A sufficient condition for $(\mathcal{A})$ to hold is that at least one of the three curves is not a "saw tooth"-like curve, similar to the one in [6, Figure 3], where all the vertex normals $\vec{\omega}_{i, j}^{m}$, $j=1 \rightarrow N_{i}-1$, are linearly dependent.

TheOREm 2.9. Let assumption $(\mathcal{A})$ hold. Then there exists a unique solution $\left\{\vec{X}^{m+1}, \kappa^{m+1}\right\} \in \underline{V}^{h} \times W_{\mathcal{M}}^{h}$ to the system $(2.30 \mathrm{a}, \mathrm{b})$.

Proof. As $(2.30 \mathrm{a}, \mathrm{b})$ is linear, existence follows from uniqueness. To investigate the latter, we consider the system: Find $\{\vec{X}, \kappa\} \in \underline{V}^{h} \times W_{\mathcal{M}}^{h}$ such that

$$
\begin{aligned}
\left\langle\vec{X}, \chi \vec{\nu}^{m}\right\rangle_{m}^{h}-\tau_{m}\langle\sigma \kappa, \chi\rangle_{m}^{h}=0 & \forall \chi \in W_{\mathcal{M}}^{h}, \\
\left\langle\kappa \vec{\nu}^{m}, \vec{\eta}\right\rangle_{m}^{h}+\left\langle\nabla_{s} \vec{X}, \nabla_{s} \vec{\eta}\right\rangle_{m}=0 & \forall \vec{\eta} \in \underline{V}^{h} .
\end{aligned}
$$

Similarly to (2.6a,b), choosing $\chi=\kappa \in W_{\mathcal{M}}^{h}$ in (2.35a) and $\vec{\eta}=\vec{X} \in \underline{V}^{h}$ in (2.35b) yields that

$$
\left\langle\nabla_{s} \vec{X}, \nabla_{s} \vec{X}\right\rangle_{m}+\tau_{m}\langle\sigma \kappa, \kappa\rangle_{m}^{h}=0 .
$$

It follows from (2.36) that $\kappa \equiv 0$ and $\vec{X} \equiv \vec{X}^{c}=\left(\vec{X}_{1}^{c}, \vec{X}_{2}^{c}, \vec{X}_{3}^{c}\right)^{T} \in\left(\mathbb{R}^{d}\right)^{3}$ with $\vec{X}_{1}^{c}=\vec{X}_{2}^{c}=\vec{X}_{3}^{c}$, and hence

$$
\left\langle\vec{X}^{c}, \chi \vec{\nu}^{m}\right\rangle_{m}^{h}=0 \quad \forall \chi \in W_{\mathcal{M}}^{h}
$$

Similarly to (2.8), choosing $\chi=\varphi_{j}^{i}$ in (2.37) and noting that $\vec{X}_{1}^{c}=\vec{X}_{2}^{c}=\vec{X}_{3}^{c}$ yields that $\vec{X}_{1}^{c} \cdot \vec{\omega}_{i, j}^{m}=0$ for all $j=1 \rightarrow N_{i}-1, i=1 \rightarrow 3$. Assumption $(\mathcal{A})$ then yields that $\vec{X}_{1}^{c}=\overrightarrow{0}$, and hence $\vec{X}^{c}=\overrightarrow{0}$. Hence we have shown that $(2.30 \mathrm{a}, \mathrm{b})$ has a unique solution $\left\{\vec{X}^{m+1}, \kappa^{m+1}\right\} \in \underline{V}^{h} \times W_{\mathcal{M}}^{h}$.

The proof immediately carries over to the system $(2.33 \mathrm{a}, \mathrm{b})$.

Furthermore, we can establish that our scheme is unconditionally stable.

THEOREM 2.10. Let $\left\{\vec{X}^{m}, \kappa^{m}\right\}_{m=1}^{M}$ be the solution of $(2.30 \mathrm{a}, \mathrm{b})$. Then for all $k=1 \rightarrow M$ we have that

$$
\left|\Gamma^{k}\right|+\sum_{m=0}^{k-1} \tau_{m}\left\langle\sigma \kappa^{m+1}, \kappa^{m+1}\right\rangle_{m}^{h} \leq\left|\Gamma^{0}\right|,
$$

where $\left|\Gamma^{k}\right|:=\int_{\Gamma^{k}} 1 \mathrm{~d} s \equiv \sum_{i=1}^{3} \sigma_{i}\left|\Gamma_{i}^{k}\right|$ on recalling the definition (2.29).

Proof. Choosing $\chi=\kappa^{m+1} \in W_{\mathcal{M}}^{h}$ in (2.30a) and $\vec{\eta}=\frac{\vec{X}^{m+1}-\vec{X}^{m}}{\tau_{m}} \in \underline{V}^{h}$ in (2.30b) yields that

$$
\left\langle\nabla_{s} \vec{X}^{m+1}, \nabla_{s}\left(\vec{X}^{m+1}-\vec{X}^{m}\right)\right\rangle_{m}+\tau_{m}\left\langle\sigma \kappa^{m+1}, \kappa^{m+1}\right\rangle_{m}^{h}=0 .
$$


We now analyze the first term in (2.39), using the ideas in [18]. Let $\vec{h}_{j}^{i, m}:=\vec{X}_{i}^{m}\left(q_{j+1}^{i}\right)-$ $\vec{X}_{i}^{m}\left(q_{j}^{i}\right)$. Then it holds that

$$
\begin{aligned}
& \left\langle\nabla_{s} \vec{X}^{m+1}, \nabla_{s}\left(\vec{X}^{m+1}-\vec{X}^{m}\right)\right\rangle_{m}=\sum_{i=1}^{3} \sigma_{i} \int_{\Gamma_{i}^{m}}\left[\nabla_{s} \vec{X}^{m+1}\right] \cdot\left[\nabla_{s}\left(\vec{X}^{m+1}-\vec{X}^{m}\right)\right] \mathrm{d} s \\
& \quad=\sum_{i=1}^{3} \sigma_{i} \sum_{j=1}^{N_{i}-1}\left[\frac{\left|\vec{h}_{j}^{i, m+1}\right|^{2}-\vec{h}_{j}^{i, m+1} \cdot \vec{h}_{j}^{i, m}}{\left|\vec{h}_{j}^{i, m}\right|}\right] \\
& \quad \geq \sum_{i=1}^{3} \sigma_{i} \sum_{j=1}^{N_{i}-1}\left[\left|\vec{h}_{j}^{i, m+1}\right|-\left|\vec{h}_{j}^{i, m}\right|\right]=\left|\Gamma^{m+1}\right|-\left|\Gamma^{m}\right| .
\end{aligned}
$$

Combining (2.39) and (2.40) yields that

$$
\left|\Gamma^{m+1}\right|-\left|\Gamma^{m}\right|+\tau_{m} \int_{\Gamma^{m}} \sigma\left|\kappa^{m+1}\right|^{2} \mathrm{~d} s \leq 0 .
$$

Summing (2.41) for $m=0 \rightarrow k-1$ yields the desired result.

The proof above is written explicitly for $(2.30 \mathrm{a}, \mathrm{b})$, but as it depends solely on a specific choice of test functions, it immediately carries over to $(2.33 \mathrm{a}, \mathrm{b})$, as well as $(2.3 \mathrm{a}, \mathrm{b})$ with the changes, in the case of nonlinear $f$, stated after Theorem 2.3.

Let $N:=\sum_{i=1}^{3}\left(N_{i}+1\right)$. We define the orthogonal projection $\vec{P}:\left(\mathbb{R}^{d}\right)^{N} \rightarrow$ $\underline{\mathbb{X}}:=\left\{\left(\vec{z}_{1}, \vec{z}_{2}, \vec{z}_{3}\right) \in\left(\mathbb{R}^{d}\right)^{N}:\left[\vec{z}_{1}\right]_{0}=\left[\vec{z}_{2}\right]_{0}=\left[\vec{z}_{3}\right]_{0},\left[\vec{z}_{1}\right]_{N_{1}}=\left[\vec{z}_{2}\right]_{N_{2}}=\left[\vec{z}_{3}\right]_{N_{3}}\right\}$ onto the Euclidean space associated with $\underline{V}^{h}$.

In order to give a matrix formulation for $(2.30 \mathrm{a}, \mathrm{b})$ we introduce the matrices $M^{i} \in \mathbb{R}^{\left(N_{i}+1\right) \times\left(N_{i}+1\right)}, \vec{N}^{i} \in\left(\mathbb{R}^{d}\right)^{\left(N_{i}+1\right) \times\left(N_{i}+1\right)}, A^{i} \in \mathbb{R}^{\left(N_{i}+1\right) \times\left(N_{i}+1\right)}$, and $\vec{A}^{i} \in$ $\left(\mathbb{R}^{d \times d}\right)^{\left(N_{i}+1\right) \times\left(N_{i}+1\right)}, i=1 \rightarrow 3$, defined by

$$
\begin{aligned}
M_{k l}^{i} & :=\sigma_{i} \int_{\Gamma_{i}^{m}} \pi_{i}^{h}\left[\phi_{k}^{i} \phi_{l}^{i}\right] \mathrm{d} s, & \vec{N}_{k l}^{i}:=\sigma_{i} \int_{\Gamma_{i}^{m}} \pi_{i}^{h}\left[\phi_{k}^{i} \phi_{l}^{i}\right] \vec{\nu}^{m} \mathrm{~d} s, \\
A_{k l}^{i} & :=\sigma_{i} \int_{\Gamma_{i}^{m}} \nabla_{s} \phi_{k}^{i} \cdot \nabla_{s} \phi_{l}^{i} \mathrm{~d} s, & \vec{A}_{k l}^{i}:=A_{k l}^{i} \overrightarrow{I d}_{1},
\end{aligned}
$$

where $\left\{\phi_{l}^{i}\right\}_{l=0}^{N_{i}}$ is the standard basis of $S_{i}^{h}:=\left\{\chi \in C(I, \mathbb{R}):\left.\chi\right|_{I_{j}^{i}}\right.$ is linear $\forall j=$ $\left.1 \rightarrow N_{i}\right\}$ and $\pi_{i}^{h}: C(I, \mathbb{R}) \rightarrow S_{i}^{h}$ is the standard interpolation operator at the nodes $\left\{q_{j}^{i}\right\}_{j=0}^{N_{i}}$. Then on introducing the matrices

$$
M:=\left(\begin{array}{ccc}
\sigma_{1} M^{1} & 0 & 0 \\
0 & \sigma_{2} M^{2} & 0 \\
0 & 0 & \sigma_{3} M^{3}
\end{array}\right), \vec{A}:=\left(\begin{array}{ccc}
\vec{A}^{1} & 0 & 0 \\
0 & \vec{A}^{2} & 0 \\
0 & 0 & \vec{A}^{3}
\end{array}\right), \vec{N}:=\left(\begin{array}{ccc}
\vec{N}^{1} & 0 & 0 \\
0 & \vec{N}^{2} & 0 \\
0 & 0 & \vec{N}^{3}
\end{array}\right),
$$

where $M: \mathbb{R}^{N} \rightarrow \mathbb{R}^{N}, \vec{A}:\left(\mathbb{R}^{d}\right)^{N} \rightarrow\left(\mathbb{R}^{d}\right)^{N}$, and $\vec{N}: \mathbb{R}^{N} \rightarrow\left(\mathbb{R}^{d}\right)^{N}$, the system of equations $(2.30 \mathrm{a}, \mathrm{b})$ can be written as: Find $\left\{\delta \vec{X}^{m+1}, \kappa^{m+1}\right\} \in \underline{\mathbb{X}} \times \mathbb{R}^{N}$ such that

$$
\left(\begin{array}{cc}
\tau_{m} M & -\vec{N}^{T} \vec{P} \\
\vec{P} \vec{N} & \vec{P} \vec{A} \vec{P}
\end{array}\right)\left(\begin{array}{c}
\kappa^{m+1} \\
\delta \vec{X}^{m+1}
\end{array}\right)=\left(\begin{array}{c}
0 \\
-\vec{P} \vec{A} \vec{P} \vec{X}^{m}
\end{array}\right)
$$

Here, with an obvious abuse of notation as in $(2.12), \kappa^{m+1}=\left(\kappa_{1}^{m+1}, \kappa_{2}^{m+1}, \kappa_{3}^{m+1}\right)^{T}$ with $\kappa_{i}^{m+1}=\left(\left[\kappa_{i}^{m+1}\right]_{0}, \ldots,\left[\kappa_{i}^{m+1}\right]_{N_{i}}\right), i=1 \rightarrow 3$, and $\delta \vec{X}^{m+1}=\left(\delta \vec{X}_{1}^{m+1}, \delta \vec{X}_{2}^{m+1}\right.$, 
$\left.\delta \vec{X}_{3}^{m+1}\right)^{T}$ with $\delta \vec{X}_{i}^{m+1}=\left(\left[\delta \vec{X}_{i}^{m+1}\right]_{0}, \ldots,\left[\delta \vec{X}_{i}^{m+1}\right]_{N_{i}}\right), i=1 \rightarrow 3$, are the vectors of coefficients with respect to the standard basis $\left\{\left\{\phi_{l}^{i}\right\}_{l=0}^{N_{i}}\right\}_{i=1}^{3}$ of $\kappa^{m+1}$ and $\vec{X}^{m+1}-\vec{X}^{m}$ in $(2.30 \mathrm{a}, \mathrm{b})$, respectively.

As $M$ is nonsingular, we can reformulate (2.44), similarly to (2.13a,b), as

$$
\begin{aligned}
\kappa^{m+1} & =\frac{1}{\tau_{m}} M^{-1} \vec{N}^{T} \vec{P} \delta \vec{X}^{m+1}, \\
\left(\vec{P} \vec{A} \vec{P}+\frac{1}{\tau_{m}} \vec{P} \vec{N} M^{-1} \vec{N}^{T} \vec{P}\right) \delta \vec{X}^{m+1} & =-\vec{P} \vec{A} \vec{P} \vec{X}^{m}
\end{aligned}
$$

TheOREM 2.11. Let $\left\{\delta \vec{X}^{m+1}, \kappa^{m+1}\right\} \in \mathbb{X} \times \mathbb{R}^{N}$ be the unique solution to (2.44). Then $\delta \vec{X}^{m+1}$ uniquely solves (2.45b). Moreover, the operator in (2.45b) is symmetric positive definite.

Proof. The proof is straightforward.

The system $(2.45 \mathrm{a}, \mathrm{b})$ is easily adapted to cover the approximation $(2.33 \mathrm{a}, \mathrm{b})$ of the setup displayed in Figure 2. In particular, we need to introduce the orthogonal projection $\vec{P}_{\partial}:\left(\mathbb{R}^{d}\right)^{N} \rightarrow \underline{\mathbb{X}}_{\partial}:=\left\{\left(\vec{z}_{1}, \vec{z}_{2}, \vec{z}_{3}\right) \in\left(\mathbb{R}^{d}\right)^{N}:\left[\vec{z}_{1}\right]_{0}=\left[\vec{z}_{2}\right]_{0}=\right.$ $\left[\vec{z}_{3}\right]_{0}$ and $\left.\left[\vec{z}_{i}\right]_{N_{i}} . \nabla F\left(\left[\vec{X}_{i}^{m}\right]_{N_{i}}\right)=0 \forall i=1 \rightarrow 3\right\}$ onto the Euclidean spaces associated with $\underline{V}_{\partial}^{h}\left(\vec{X}^{m}\right)$. Then $(2.45 \mathrm{~b})$ is readily replaced by

$$
\left(\vec{P}_{\partial} \vec{A} \vec{P}_{\partial}+\frac{1}{\tau_{m}} \vec{P}_{\partial} \vec{N} M^{-1} \vec{N}^{T} \vec{P}_{\partial}\right) \delta \vec{X}^{m+1}=-\vec{P}_{\partial} \vec{A} \vec{X}^{m} .
$$

We note that (2.13b) and (2.46) can easily be generalized to curve networks with an arbitrary number of triple junctions and external boundary intersections; see Remark 2.13 below for the relevant details.

2.2.1. Surface diffusion of curve networks. We recall the following approximation to (1.19), with $(1.14 \mathrm{a}, \mathrm{b})$ and $(1.21 \mathrm{a}, \mathrm{b})$, as proposed in [6]: Find $\left\{\vec{X}^{m+1}, \kappa^{m+1}\right\}$ $\in \underline{V}^{h} \times W^{h}$ such that

$$
\begin{aligned}
\left\langle\frac{\vec{X}^{m+1}-\vec{X}^{m}}{\tau_{m}}, \chi \vec{\nu}^{m}\right\rangle_{m}^{h}-\left\langle\sigma \nabla_{s} \kappa^{m+1}, \nabla_{s} \chi\right\rangle_{m}=0 & \forall \chi \in W^{h}, \\
\left\langle\kappa^{m+1} \vec{\nu}^{m}, \vec{\eta}\right\rangle_{m}^{h}+\left\langle\nabla_{s} \vec{X}^{m+1}, \nabla_{s} \vec{\eta}\right\rangle_{m}=0 & \forall \vec{\eta} \in \underline{V}^{h},
\end{aligned}
$$

where

$$
W^{h}:=\left\{\left(\chi_{1}, \chi_{2}, \chi_{3}\right) \in W:\left.\chi_{i}\right|_{I_{j}^{i}} \text { is linear } \forall j=1 \rightarrow N_{i}, i=1 \rightarrow 3\right\} \subset W
$$

and $W:=\left\{\left(\chi_{1}, \chi_{2}, \chi_{3}\right) \in[C(I, \mathbb{R})]^{3}: \sigma_{1} \chi_{1}+\sigma_{2} \chi_{2}+\sigma_{3} \chi_{3}=0\right.$ on $\left.\partial I\right\}$. We recall further that the system $(2.47 \mathrm{a}, \mathrm{b})$ can be solved by applying a Schur complement approach and then solving for $\delta \vec{X}^{m+1} \in \underline{\mathbb{X}}$ :

$$
\vec{\Pi} \vec{P}\left(\vec{A}+\frac{1}{\tau_{m}} \vec{N} K S K \vec{N}^{T}\right) \vec{P} \vec{\Pi} \delta \vec{X}^{m+1}=-\vec{\Pi} \vec{P} \vec{A} \vec{P} \vec{X}^{m}
$$

Here $K: \mathbb{R}^{N} \rightarrow \mathbb{X}:=\left\{\left(z_{1}, z_{2}, z_{3}\right) \in \mathbb{R}^{N}: \sum_{i=1}^{3} \sigma_{i}\left[z_{i}\right]_{0}=\sum_{i=1}^{3} \sigma_{i}\left[z_{i}\right]_{N_{i}}=0\right\}$ is the orthogonal projection onto $\mathbb{X}$, and $S$ is the inverse of $K A K$, where $A$ is defined similarly to (2.43) using $A^{i}$ in $(2.42)$, on the space $(\operatorname{ker} K A K)^{\perp}$. Also $\vec{\Pi}:\left(\mathbb{R}^{d}\right)^{N} \rightarrow \mathcal{R}^{\perp}$ is the orthogonal projection onto $\mathcal{R}^{\perp}$, where $\mathcal{R}:=\operatorname{span}\left\{\vec{P} \vec{N} K e_{i}: i=1 \rightarrow 2\right\} \equiv$ 
$\{\vec{P} \vec{N} K v: v \in \operatorname{ker} K A K\} \subset \underline{\mathbb{X}}$ with $\left\{e_{i}\right\}_{i=1}^{2}, e_{1}:=\left(\frac{1}{\sigma_{1}} 1^{1},-\frac{1}{\sigma_{2}} 1^{2}, 0\right) \in \mathbb{X}$ and $e_{2}:=$ $\left(0, \frac{1}{\sigma_{2}} 1^{2},-\frac{1}{\sigma_{3}} 1^{3}\right) \in \mathbb{X}$, where $1^{i}:=(1, \ldots, 1)^{T} \in \mathbb{R}^{N_{i}+1}, i=1 \rightarrow 3$, being a basis of the space $E=\operatorname{ker} A \cap \mathbb{X}$.

We now want to adapt the above scheme to include possible boundary intersections. Naturally in the case of $(1.17 \mathrm{~b}),(2.47 \mathrm{a}, \mathrm{b})$ is changed to: Find $\left\{\delta \vec{X}^{m+1}, \kappa^{m+1}\right\} \in$ $\underline{V}_{\partial}^{h}\left(\vec{X}^{m}\right) \times W_{\partial}^{h}$ such that

$$
\begin{aligned}
& \left\langle\frac{\delta \vec{X}^{m+1}}{\tau_{m}}, \chi \vec{\nu}^{m}\right\rangle_{m}^{h}-\left\langle\sigma \nabla_{s} \kappa^{m+1}, \nabla_{s} \chi\right\rangle_{m}=0 \quad \forall \chi \in W_{\partial}^{h}, \\
& \left\langle\kappa^{m+1} \vec{\nu}^{m}, \vec{\eta}\right\rangle_{m}^{h}+\left\langle\nabla_{s} \vec{X}^{m+1}, \nabla_{s} \vec{\eta}\right\rangle_{m}=0 \quad \forall \vec{\eta} \in \underline{V}_{\partial}^{h}\left(\vec{X}^{m}\right),
\end{aligned}
$$

where $W_{\partial}:=\left\{\left(\chi_{1}, \chi_{2}, \chi_{3}\right) \in[C(I, \mathbb{R})]^{3}: \sigma_{1} \chi_{1}(0)+\sigma_{2} \chi_{2}(0)+\sigma_{3} \chi_{3}(0)=0\right\}$ and $W_{\partial}^{h}$ is defined similarly to (2.48). On defining the orthogonal projection $K_{\partial}: \mathbb{R}^{N} \rightarrow \mathbb{X}_{\partial}:=$ $\left\{\left(z_{1}, z_{2}, z_{3}\right) \in \mathbb{R}^{N}: \sum_{i=1}^{3} \sigma_{i}\left[z_{i}\right]_{0}=0\right\}$ and using the projection $\vec{P}_{\partial}$ defined earlier, we can apply a Schur complement approach to yield

$$
\vec{\Pi}_{\partial} \vec{P}_{\partial}\left(\vec{A}+\frac{1}{\tau_{m}} \vec{N} K_{\partial} S_{\partial} K_{\partial} \vec{N}^{T}\right) \vec{P}_{\partial} \vec{\Pi}_{\partial} \delta \vec{X}^{m+1}=-\vec{\Pi}_{\partial} \vec{P}_{\partial} \vec{A} \vec{X}^{m}
$$

where $S_{\partial}$ is the inverse of $K_{\partial} A K_{\partial}$ on the space $\left(\operatorname{ker} K_{\partial} A K_{\partial}\right)^{\perp}$ and $\vec{\Pi}_{\partial}$ is the orthogonal projection from $\mathbb{R}^{N}$ onto $\mathcal{R}_{\partial}^{\perp}$ with $\mathcal{R}_{\partial}:=\vec{P}_{\partial} \vec{N} K_{\partial}\left(\operatorname{ker} K_{\partial} A K_{\partial}\right)$. In fact, for the setup in Figure 2, $\vec{\Pi}_{\partial} \equiv \vec{\Pi}$. However, in general this is not always the case; see Remark 2.14 below. Of course, the above is extended to (1.18) by replacing (2.50b) by $(2.34)$.

Remark 2.12. A possible definition for the projection $\vec{\Pi}$ is $\vec{\Pi}:=\overrightarrow{I d}_{N}-\vec{Q} \vec{Q}^{T}$, where $\operatorname{im} \vec{Q}=\mathcal{R}$ and $\vec{Q}^{T} \vec{Q}=I d_{2}$. In other words, the columns of $\vec{Q} \in\left(\mathbb{R}^{d}\right)^{N \times 2}$ are an orthonormal basis of the subspace $\mathcal{R} \subset\left(\mathbb{R}^{d}\right)^{N}$ spanned by $\vec{P} \vec{N} K e_{i} \equiv \vec{P} \vec{N} e_{i}$, where $e_{i} \in \mathbb{X}, i=1 \rightarrow 2$, are the above mentioned null vectors of $K A K$. We note that the definitions of $\vec{P}$ and $\vec{N}$ yield that $\operatorname{dim} \mathcal{R}=2$. Hence $\vec{\Pi}$ is the orthogonal projection from $\left(\mathbb{R}^{d}\right)^{N}$ onto $(\operatorname{im} \vec{Q})^{\perp} \equiv \mathcal{R}^{\perp}$.

Remark 2.13. The definitions of the spaces $\underline{V}$ and $W$ can easily be generalized to a situation with $K_{B}$ bubbles (enclosed areas), $K_{C}$ curves, and $K_{T}$ triple junction points. Note that Euler's formula yields that $6\left(K_{B}-1\right)=2 K_{C}=3 K_{T}$ in the absence of external boundary intersections. For example, $K_{B}=2, K_{C}=3$, and $K_{T}=2$ in Figure 1. In particular, we would have that $\underline{V}:=\left\{\left(\chi_{1}, \ldots, \chi_{K_{C}}\right) \in\left[C\left(I, \mathbb{R}^{d}\right)\right]^{K_{C}}\right.$ : $\left.\chi_{i_{j}}\left(p_{j, i_{j}}\right)=\chi_{1_{j}}\left(p_{j, 1_{j}}\right), i=2 \rightarrow 3, \forall j=1 \rightarrow \overline{K_{T}}\right\}$ and

$W:=\left\{\left(\chi_{1}, \ldots, \chi_{K_{C}}\right) \in[C(I, \mathbb{R})]^{K_{C}}: \sum_{i=1}^{3}(-1)^{p_{j, i_{j}}} \sigma_{i_{j}} \chi_{i_{j}}\left(p_{j, i_{j}}\right)=0 \quad \forall j=1 \rightarrow K_{T}\right\}$.

Here $i_{j} \in\left\{1, \ldots, K_{C}\right\}, i=1 \rightarrow 3$, denotes the three curves meeting at triple junction $j$, while $p_{j, i_{j}} \in\{0,1\}$ denotes whether these curves start $\left(p_{j, i_{j}}=0\right)$ or end $\left(p_{j, i_{j}}=1\right)$ at the triple junction point $j$. That is, $\left|\left\{i_{j}: i=1 \rightarrow 3\right\}\right|=3$ for all $j=1 \rightarrow K_{T}$, $\left|\left\{j: i_{j}=c\right\}\right|=2$ for all $c=1 \rightarrow K_{C}$, and $\sum_{j=1}^{K_{T}} p_{j, c}=1$ for all $c=1 \rightarrow K_{C}$.

The above definitions are easily generalized to the possible presence of external boundary intersections. Let $K_{B}$ denote the number of bubbles (enclosed areas), $K_{T}$ the number of triple junctions, $K_{C}$ the number of curves, and $K_{I}$ the number of 
intersections with the external boundary. Then Euler's formula yields that $6\left(K_{B}-\right.$ $1)=2\left(K_{C}+K_{I}\right)=3\left(K_{T}+K_{I}\right)$. (Note that the special case $K_{I}=0$ corresponds to the formula given above.) For example, $K_{B}=3, K_{C}=3, K_{T}=1$, and $K_{I}=3$ in Figure 2. The corresponding precise definitions of the spaces $\underline{V}_{\partial}\left(\vec{X}^{m}\right)$ and $W_{\partial}$ are straightforward, and we omit the details here.

Remark 2.14. The definition of $\vec{\Pi}$ can easily be adapted to a situation with $K_{B}$ bubbles. In the case of no external boundary intersections, the subspace $E:=\mathbb{X} \cap \operatorname{ker} A$ of the kernel of $K A K$ has dimension $K_{B}$, and a possible basis consists of vectors that each "describe an admissible orientation of the boundary of a bubble" in terms of the given $K_{C}$ curves. For example, if $K_{B}=3$, one area is enclosed by curves $1,2,4$, and curve 2 is parameterized in the opposite direction to curves 1 and 4 , then the corresponding eigenvector would be $\left(\frac{1}{\sigma_{1}} 1^{1},-\frac{1}{\sigma_{2}} 1^{2}, 0, \frac{1}{\sigma_{4}} 1^{4}, 0,0\right)$. See the appendix in [6] for a more rigorous definition.

When boundary intersections are present, the subspace $E$ has dimension $K_{B}-1$, and a possible basis consists of vectors that each "describe an admissible orientation of the boundary of a bubble" (ignoring the boundary part of any bubble that is made up by the external boundary $\partial \Omega$ ) in terms of the given $K_{C}$ curves. Similarly to [6, Appendix] one can show that it indeed holds that $\operatorname{dim} E=K_{C}-K_{T}=\left(K_{C}+K_{I}\right)-$ $\left(K_{T}+K_{I}\right)=3\left(K_{B}-1\right)-2\left(K_{B}-1\right)=K_{B}-1$.

One can interpret each set of basis vectors of $E$ as a linearly independent collection of regions that preserve their areas. Then it is intuitive that the respective dimensions of $E$ in the two described cases are $K_{B}$ and $K_{B}-1$.

2.2.2. Combined surface diffusion and mean curvature flow. We now adapt $(2.47 \mathrm{a}, \mathrm{b})$ so that it approximates the evolution law $(1.23)$, with $(1.14 \mathrm{a}, \mathrm{b})$ and $(1.24 \mathrm{a}, \mathrm{b})$. Choosing $\underline{V}^{h}$ based on $\underline{V}$, as defined in Remark 2.13, and $W_{\star}^{h}$ based on $($ recall $(2.52)) W_{\star}:=\left\{\left(\chi_{1}, \ldots, \chi_{K_{C}}\right) \in[C(I, \mathbb{R})]^{K_{C}}: \sum_{i=1}^{3} \mathcal{H}_{\left\{i_{0}+1 \rightarrow K_{C}\right\}}\left(i_{j}\right)\left[(-1)^{p_{j, i_{j}}}\right.\right.$ $\left.\left.\sigma_{i_{j}} \chi_{i_{j}}\left(p_{j, i_{j}}\right)\right]=0 \forall j=1 \rightarrow K_{T}\right\}$, where $\mathcal{H}_{\mathcal{F}}$ is the indicator function for the set $\mathcal{F}$, yields the following approximation: Find $\left\{\vec{X}^{m+1}, \kappa^{m+1}\right\} \in \underline{V}^{h} \times W_{\star}^{h}$ such that

$$
\begin{aligned}
\left\langle\frac{\vec{X}^{m+1}-\vec{X}^{m}}{\tau_{m}}, \chi \vec{\nu}^{m}\right\rangle_{m}^{h}-\left\langle\sigma \kappa^{m+1}, \chi\right\rangle_{m}^{\star}=0 & \forall \chi \in W_{\star}^{h}, \\
\left\langle\kappa^{m+1} \vec{\nu}^{m}, \vec{\eta}\right\rangle_{m}^{h}+\left\langle\nabla_{s} \vec{X}^{m+1}, \nabla_{s} \vec{\eta}\right\rangle_{m}=0 & \forall \vec{\eta} \in \underline{V}^{h},
\end{aligned}
$$

where $\langle\sigma \eta, \chi\rangle_{m}^{\star}:=\left\langle\left.\sigma \eta\right|_{\Gamma^{m, M C}},\left.\chi\right|_{\Gamma^{m, M C}}\right\rangle_{m}^{h}+\left\langle\left.\sigma \nabla_{s} \eta\right|_{\Gamma^{m, S D}},\left.\nabla_{s} \chi\right|_{\Gamma^{m, S D}}\right\rangle_{m}$ for all $\eta, \chi \in W_{\star}^{h}$. Here $\Gamma^{m, M C}:=\bigcup_{i=1}^{i_{0}} \Gamma_{i}^{m}$ and $\Gamma^{m, S D}:=\bigcup_{i=i_{0}+1}^{K_{C}} \Gamma_{i}^{m}$. The above (weakly) approximates the correct conditions at the triple junctions, (1.14a,b) and (1.24a,b). Once again, it is a straightforward matter to derive an existence and uniqueness result and a stability result for $(2.53 \mathrm{a}, \mathrm{b})$ under an appropriate mild assumption.

Using the natural extensions from the setup in Figure 1 to $K_{C}$ curves of the matrices defined earlier, recalling (2.42) and (2.43), introducing $A_{\star}:=\operatorname{diag}\left(\sigma_{1} M^{1}, \ldots\right.$, $\sigma_{i_{0}} M^{i_{0}}, \sigma_{i_{0}+1} A^{i_{0}+1}, \ldots, \sigma_{K_{C}} A^{K_{C}}$ ), extending the associated operators, and introducing the orthogonal projection $K_{\star}$ onto the Euclidean space $\mathbb{X}_{\star}$ associated with $W_{\star}^{h}$, we can derive the following equations for the extended coefficient vectors:

$$
\left(\begin{array}{cc}
\tau_{m} K_{\star} A_{\star} K_{\star} & -K_{\star} \vec{N}^{T} \vec{P} \\
\vec{P} \vec{N} K_{\star} & \vec{P} \vec{A} \vec{P}
\end{array}\right)\left(\begin{array}{c}
\kappa^{m+1} \\
\delta \vec{X}^{m+1}
\end{array}\right)=\left(\begin{array}{c}
0 \\
-\vec{P} \vec{A} \vec{P} \vec{X}^{m}
\end{array}\right) .
$$

Introducing the inverse $S_{\star}$ of $K_{\star} A_{\star} K_{\star}$ restricted on the set $\left(\operatorname{ker} K_{\star} A_{\star} K_{\star}\right)^{\perp}=\mathbb{X}_{\star} \cap$ ker $A_{\star}$, i.e., $S_{\star} K_{\star} A_{\star} K_{\star} v=K_{\star} A_{\star} K_{\star} S_{\star} v=v$ for all $v \in \mathbb{X}_{\star} \cap \operatorname{ker} A_{\star}$, we can employ 
a Schur complement approach in order to transform (2.54) to

$$
\begin{aligned}
& \kappa^{m+1}=\frac{1}{\tau_{m}} S_{\star} K_{\star} \vec{N}^{T} \vec{P} \delta \vec{X}^{m+1}+z, \quad z \in \mathbb{X}_{\star} \cap \operatorname{ker} A_{\star}, \\
& \vec{\Pi}_{\star}\left(\vec{P} \vec{A} \vec{P}+\frac{1}{\tau_{m}} \vec{P} \vec{N} K_{\star} S_{\star} K_{\star} \vec{N}^{T} \vec{P}\right) \vec{\Pi}_{\star} \delta \vec{X}^{m+1}=-\vec{\Pi}_{\star} \vec{P} \vec{A} \vec{P} \vec{X}^{m},
\end{aligned}
$$

where $\vec{\Pi}_{\star}:\left(\mathbb{R}^{d}\right)^{N} \rightarrow \mathcal{R}_{\star}^{\perp}$ is the orthogonal projection onto $\mathcal{R}_{\star}^{\perp}$ with $\mathcal{R}_{\star}:=\vec{P} \vec{N} K_{\star} E_{\star}$ and $E_{\star}:=\mathbb{X}_{\star} \cap \operatorname{ker} A_{\star}$.

Remark 2.15. A basis of $\mathcal{R}_{\star}$ can be found similarly to the pure surface diffusion case; see Remark 2.14. The corresponding basis of $E_{\star}$ is now made up of vectors that assign orientations for bubble boundaries to linear independent collections of curves $\Gamma_{i}^{m}$ with $i>i_{0}$. Of course, one can extend the approximation $(2.53 \mathrm{a}, \mathrm{b})$ to include external boundaries in the natural way. Although we omit the details here, some numerical results are given in section 3 .

2.3. Quadruple junctions. A further generalization of the schemes $(2.33 \mathrm{a}, \mathrm{b})$, $(2.50 \mathrm{a}, \mathrm{b})$, and $(2.53 \mathrm{a}, \mathrm{b})$ in this paper is the extension from triple junction points to quadruple junction points. This needs only a minor change to the introduced finite element spaces, and we omit the exact details here. However, numerical results for this practically interesting situation can be found in the next section.

3. Results. The Schur complement systems (2.13b), (2.26c), (2.45b), (2.46), (2.49), (2.51), and (2.55b) can be easily solved with a conjugate gradient solver. Where necessary, the solution of $K A K y=x$ and its variants in order to compute $S x, S_{\partial} x, S_{\star} x$, and $S_{0} x$, respectively, can be obtained with an (inner loop) CG solver without a projection, as the right-hand-side vector $x$ always satisfies the necessary compatibility condition, e.g., $x \in(\operatorname{ker} K A K)^{\perp}$. See [30] for a justification for using a CG solver for a positive semidefinite system.

The system (2.14b), on the other hand, can be solved with an inexact Newton method. When $f$ is given by (1.6), because of the singularity of $f^{-1}=f$ at the origin, the discrete system $(2.14 \mathrm{~b})$ needs to be solved with a damped inexact Newton method, where as initial guess for the Newton iteration we choose $\delta \vec{X}^{m+1,0}:=\vec{N}_{0} 1$. Moreover, we perform computations only for (1.7) with (1.6), where the evolution is well defined for all times, e.g., where the initial data $\vec{x}(\cdot, 0)$ is such that that $\varkappa(\cdot, 0)>0$. In practice, the damped Newton method always converged in these cases and we always observed that $\kappa^{m}>0, m=0 \rightarrow M$. In addition, for the scheme (2.4), (2.3b) we will use the initial data $\kappa^{0}:=-\left(\vec{N}_{0}^{T} \vec{N}_{0}\right)^{-1} \vec{N}_{0}^{T} \vec{A}_{0} \vec{X}^{0}$, on noting that $\vec{N}_{0}^{T} \vec{N}_{0}$ is a diagonal matrix with strictly positive diagonal entries.

Throughout this section we use uniform time steps $\tau_{m}=\tau, m=0 \rightarrow M-1$. For later purposes, we define $\vec{X}(t):=\frac{t-t_{m-1}}{\tau} \vec{X}^{m}+\frac{t_{m}-t}{\tau} \vec{X}^{m-1}$ for $t \in\left[t_{m-1}, t_{m}\right], m \geq 1$.

3.1. Closed curves. Here we compare our scheme (2.3a,b) with $f(r):=r$, i.e., (2.17), with two other algorithms in the literature, namely the schemes (2.15) from [17] and (2.18) from [13]. As a first test, we repeat the computations for a true solution as given in $[17$, p. 604]. An exact solution to (1.7) with $f(r):=r$, so that the resulting $\Gamma(\cdot)$ solves $(1.3)$, is given by

$$
\vec{x}(\rho, t)=(1-2 t)^{\frac{1}{2}}(\cos g(\rho), \sin g(\rho))^{T}, \quad \varkappa(\rho, t)=(1-2 t)^{-\frac{1}{2}}, \quad t \in[0, \bar{T}), \bar{T}=0.5,
$$


TABLE 1

Absolute errors $\|\vec{X}-\vec{x}\|_{L^{\infty}}$ for $T=\frac{1}{2} \bar{T}=0.25$ and $T=\bar{T}-\tau$, respectively.

\begin{tabular}{|r|c|c|c|c|c|c|}
\hline \multirow{2}{*}{$N$} & $T=\frac{1}{2} \bar{T}$ & $T=\bar{T}-\tau$ & $T=\frac{1}{2} \bar{T}$ & $T=\bar{T}-\tau$ & $T=\frac{1}{2} \bar{T}$ & $T=\bar{T}-\tau$ \\
\hline 16 & $3.9879 \mathrm{e}-02$ & $1.3476 \mathrm{e}-01$ & $4.2132 \mathrm{e}-02$ & $1.3978 \mathrm{e}-01$ & $3.1574 \mathrm{e}-02$ & $1.1731 \mathrm{e}-01$ \\
32 & $1.2994 \mathrm{e}-02$ & $1.2155 \mathrm{e}-01$ & $1.3973 \mathrm{e}-02$ & $1.2496 \mathrm{e}-01$ & $1.0287 \mathrm{e}-02$ & $1.1149 \mathrm{e}-01$ \\
64 & $3.4556 \mathrm{e}-03$ & $8.4151 \mathrm{e}-02$ & $3.7408 \mathrm{e}-03$ & $8.5576 \mathrm{e}-02$ & $2.7043 \mathrm{e}-03$ & $7.8982 \mathrm{e}-02$ \\
128 & $8.7924 \mathrm{e}-04$ & $5.3324 \mathrm{e}-02$ & $9.5359 \mathrm{e}-04$ & $5.3825 \mathrm{e}-02$ & $6.8469 \mathrm{e}-04$ & $5.0839 \mathrm{e}-02$ \\
256 & $2.2112 \mathrm{e}-04$ & $3.1728 \mathrm{e}-02$ & $2.3963 \mathrm{e}-04$ & $3.1887 \mathrm{e}-02$ & $1.7184 \mathrm{e}-04$ & $3.0545 \mathrm{e}-02$ \\
512 & $5.5339 \mathrm{e}-05$ & $1.8217 \mathrm{e}-02$ & $5.6193 \mathrm{e}-05$ & $1.8270 \mathrm{e}-02$ & $4.2953 \mathrm{e}-05$ & $1.7655 \mathrm{e}-02$ \\
1024 & $1.3846 \mathrm{e}-05$ & $1.0227 \mathrm{e}-02$ & $1.4049 \mathrm{e}-05$ & $1.0245 \mathrm{e}-02$ & $1.0735 \mathrm{e}-05$ & $9.9610 \mathrm{e}-03$ \\
\hline
\end{tabular}
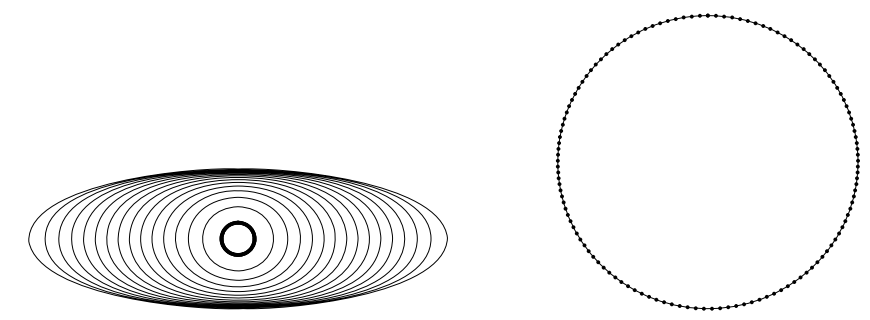

FiG. 3. A plot of $\vec{X}(t)$ at times $t=0,0.1, \ldots, 1.5$ and at time $t=T$ (scaled).
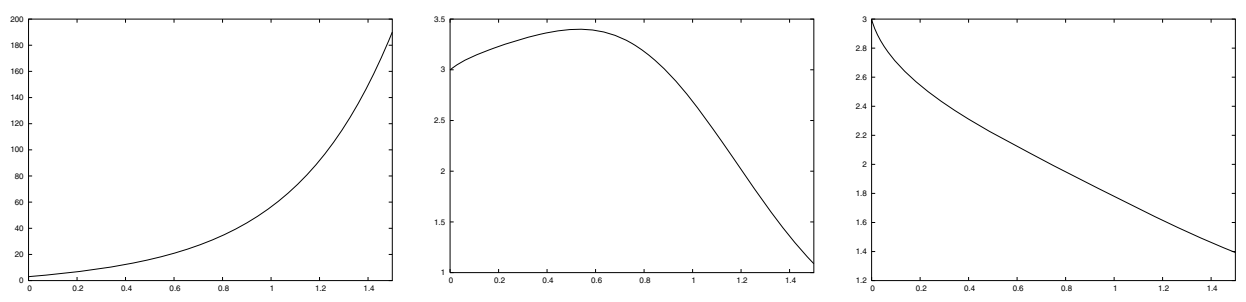

FIG. 4. A plot of the ratio $r=h_{\vec{X}^{m}} / \ell_{\vec{X}^{m}}$ for the three schemes (2.15), (2.18), and (2.17).

where $g(\rho)=2 \pi \rho+0.1 \sin (2 \pi \rho)$ in order to make the initial distribution of nodes nonuniform. We note that $\vec{x}_{t} \cdot \vec{\tau}=0$ for the solution (3.1). We compare our results from (2.17) to the schemes (2.15) and (2.18); see Table 1. We use $\tau=0.5 h^{2}$ and either $T=\frac{1}{2} \bar{T}$ or $T=\bar{T}-\tau$. Here and in what follows we always compute the error $\|\vec{X}-\vec{x}\|_{L^{\infty}}:=\max _{m=1 \rightarrow M}\left\|\vec{X}\left(t_{m}\right)-\vec{x}\left(\cdot, t_{m}\right)\right\|_{L^{\infty}}$, where $\left\|\vec{X}\left(t_{m}\right)-\vec{x}\left(\cdot, t_{m}\right)\right\|_{L^{\infty}}:=$ $\max _{j=1 \rightarrow N} \min _{\rho \in J}\left|\vec{X}^{m}\left(q_{j}\right)-\vec{x}\left(\rho, t_{m}\right)\right|$ between $\vec{X}$ and the true solution on the interval $[0, T]$. We note that the experiments indicate that the convergence rate for the error away from the singularity is $O\left(h^{2}\right)$, and up to the singularity at time $\bar{T}$ is of order less than $O(h)$, for all three schemes, as is to be expected.

The next experiment is for a mild ellipse. The parameters were chosen as follows. $N=128, \tau=10^{-2}, T=1.5$, and the initial curve is a $3: 1$ ellipse with a unit semiminor axis. In order to highlight one difference between the three schemes in consideration, we plot for each of them the ratio $r:=h_{\vec{X}^{m}} / \ell_{\vec{X}^{m}}$, where $h_{\vec{X}^{m}}:=$ $\max _{j=1 \rightarrow N}\left|\vec{X}^{m}\left(q_{j}\right)-\vec{X}^{m}\left(q_{j-1}\right)\right|$ and $\ell_{\vec{X}^{m}}:=\min _{j=1 \rightarrow N}\left|\vec{X}^{m}\left(q_{j}\right)-\vec{X}^{m}\left(q_{j-1}\right)\right|$, over time. The evolution of our scheme (2.17) can be seen in Figure 3. Plots of the ratio $r$ for the three schemes can be seen in Figure 4. One can clearly see that the ratio increases for scheme (2.15), while the tangential movement of vertices induced by the 

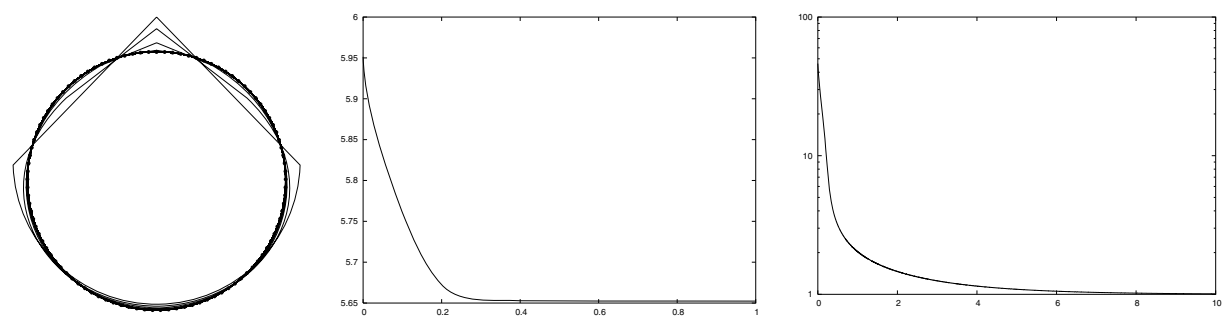

FIG. 5. Plots of $\vec{X}(t)$ at $t=0,0.1, \ldots, T=10,|\Gamma(t)|$ for $t \in[0,1]$, and $\log r(t)$ for $t \in[0, T]$.
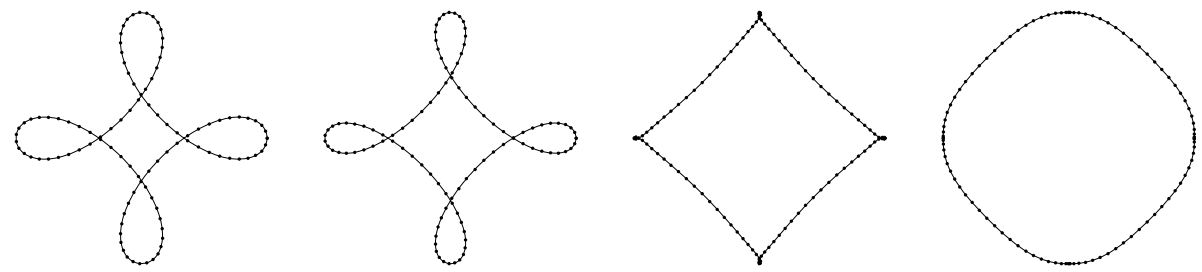

Fig. 6. Plot of $\vec{X}(t)$ at times $t=0.08,0.085,0.0885,0.9$ (scaled).

other two schemes, as discussed in Remark 2.5, results in a decrease of the ratio $r$, which approaches the optimal value 1 . In order to underline this point further, we conducted an experiment for area preserving mean curvature flow, (1.8). The initial curve for our approximation (2.4), with $f(r):=r$, and (2.3b) consists of a semicircle and a single additional node on the periphery of the circle with unit radius. We used the parameters $N=100, T=10$, and $\tau=10^{-3}$. In Figure 5 we show $\vec{X}(t)$ at different times, as well as a plot of $|\Gamma(t)|$ and $\log r(t)$ over time. One can clearly see that although an approximation to the true steady state, a circle, is reached very quickly (at around time $t=0.6$ ), in the remaining time the vertices are continually moved tangentially, which results in a further decrease in the ratio $r$ that eventually approaches the optimal value 1 .

In addition, we computed $(2.3 \mathrm{a}, \mathrm{b})$ with $f(r):=r$ for a self-intersecting curve that exhibits cusps during the evolution. The initial curve is taken from [13, Figure $1]$ and can be parameterized by $\vec{x}(\rho, 0)=\cos (4 \pi \rho)(\cos (2 \pi \rho), \sin (2 \pi \rho))^{T}$. We used the parameters $N=100, \tau=10^{-3}$, and $T=0.09$. We see in Figure 6 that for this example the mesh points are clustered in regions where the curvature is high, due to the very fast evolution there. Of course this clustering decreases as the time step size $\tau$ is reduced in line with an equidistribution result for the semidiscrete scheme; recall Remark 2.5. Similarly to other parametric algorithms, our approximation jumps across the singularity in the evolution; see, e.g., [13, Figure 1] for (2.4) and [14, Figure $4.2]$ for $(2.15)$.

For the first experiment for the nonlinear approximation $(2.3 \mathrm{a}, \mathrm{b})$ we used the exact solution to (1.4) with (1.5):

$\vec{x}(\rho, t)=(1-(\beta+1) t)^{\frac{1}{\beta+1}}(\cos g(\rho), \sin g(\rho))^{T}, \quad \varkappa(\rho, t)=(1-(\beta+1) t)^{-\frac{1}{\beta+1}}, \quad t \in[0, \bar{T})$, where $\bar{T}=\frac{1}{\beta+1}$ and $g$ is given as in (3.1). We note once again that here $\vec{x}_{t} \cdot \vec{\tau}=0$. We report on a corresponding error table for $\beta=\frac{1}{2}$ and $\beta=\frac{1}{3}$ in Table 2, where we used $\tau=0.5 h^{2}$. 
Absolute errors $\|\vec{X}-\vec{x}\|_{L^{\infty}}$ for $T=\frac{1}{2} \overline{T A B L E ~} \frac{1}{2(\beta+1)}, T=\bar{T}-\tau$, and $T=1$, respectively.

\begin{tabular}{|c|c|c|c|c|c|}
\hline \multirow[b]{2}{*}{$N$} & \multicolumn{2}{|c|}{$\beta=\frac{1}{2}$} & \multicolumn{2}{|c|}{$\beta=\frac{1}{3}$} & \multirow{2}{*}{$\begin{array}{c}f(r)=-r^{-1} \\
T=1\end{array}$} \\
\hline & $T=\frac{1}{2} \bar{T}$ & $\stackrel{2}{T}=\bar{T}-\tau$ & $T=\frac{1}{2} \bar{T}$ & $\stackrel{3}{T}=\bar{T}-\tau$ & \\
\hline 16 & $1.8812 \mathrm{e}-02$ & $8.4620 \mathrm{e}-02$ & $1.2804 \mathrm{e}-02$ & $6.0554 \mathrm{e}-02$ & $2.6054 \mathrm{e}-01$ \\
\hline 32 & $5.4838 \mathrm{e}-03$ & 6.0185 & $3.3827 \mathrm{e}-03$ & 3.890 & -02 \\
\hline 64 & $1.4210 \mathrm{e}-03$ & $3.4475 \mathrm{e}-02$ & $8.6680 \mathrm{e}-04$ & $1.9613 \mathrm{e}-02$ & $1.9709 \mathrm{e}-02$ \\
\hline 128 & $3.5637 \mathrm{e}-04$ & $1.7774 \mathrm{e}-02$ & $2.1613 \mathrm{e}-04$ & $9.0762 \mathrm{e}-03$ & $4.9869 \mathrm{e}-03$ \\
\hline 256 & $8.9173 \mathrm{e}-05$ & $8.6226 \mathrm{e}-03$ & $5.3925 \mathrm{e}-05$ & $3.9824 \mathrm{e}-03$ & $1.2502 \mathrm{e}-03$ \\
\hline 512 & $2.2226 \mathrm{e}-05$ & $4.0244 \mathrm{e}-03$ & $1.3431 \mathrm{e}-05$ & $1.6607 \mathrm{e}-03$ & $3.1255 \mathrm{e}-04$ \\
\hline
\end{tabular}
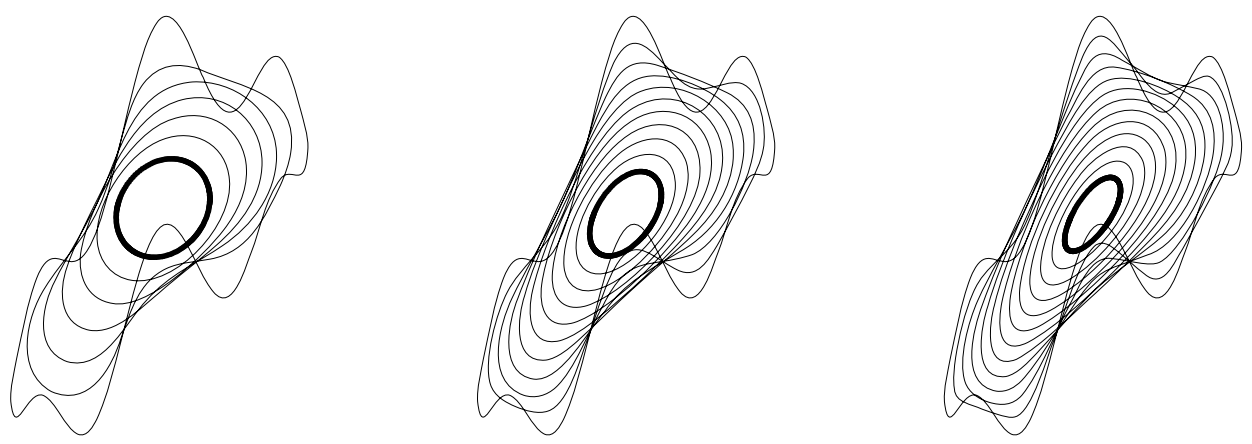

FIG. 7. $\mathcal{V}=\varkappa^{\beta}$, with $\beta=1, \frac{1}{2}, \frac{1}{3}$. We plot $\vec{X}(t)$ at times $t=0,0.05, \ldots, T$, with $T=0.3,0.5$, and 0.6 , respectively.

Next, we considered $(2.3 \mathrm{a}, \mathrm{b})$ with $(1.5)$ for the initial curve as given in [35, p. 1494] for the three different choices $\beta=1, \frac{1}{2}, \frac{1}{3}$; i.e., we chose

$$
\begin{aligned}
\vec{x}(\rho, 0)= & \left(\cos g_{0}(\rho),\right. \\
& \left.\frac{1}{2} \sin g_{0}(\rho)+\sin \left(\cos g_{0}(\rho)\right)+\sin g_{0}(\rho)\left[\frac{1}{5}+\sin g_{0}(\rho) \sin ^{2} g_{0}(3 \rho)\right]\right)^{T},
\end{aligned}
$$

where $g_{0}(\rho)=2 \pi \rho$, and set $\vec{X}^{0}=\pi^{h} \vec{x}(\cdot, 0)$ for a uniform partitioning of $J$. The numerical results for the approximation $(2.3 \mathrm{a}, \mathrm{b})$ can be seen in Figure 7 , where we used $N=256$ and $\tau=10^{-3}$. It should be noted that in [35] a system of second order PDEs is derived to model (1.4), which introduces a tangential movement that locally equidistributes nodes under discretization by a finite difference approximation. However, the system and subsequent approximation is far more complicated than our simple approximation $(2.3 \mathrm{a}, \mathrm{b})$.

3.1.1. Inverse mean curvature flow. Here we consider the flow (1.4) with (1.6). First, we performed a convergence test for the approximation $(2.3 \mathrm{a}, \mathrm{b})$ with $f$ given by (1.6). A true solution to (1.7) and (1.6) with $\vec{x}_{t} \cdot \vec{\tau}=0$ is given by

$$
\vec{x}(\rho, t)=\exp (t)(\cos g(\rho), \sin g(\rho))^{T}, \quad \varkappa(\rho, t)=\exp (-t), \quad t \in[0, \infty),
$$

where $g$ is defined as in (3.1). We report on the corresponding errors for $\tau=0.5 h^{2}$ in the last column of Table 2. The evolution for $N=256$ can be seen on the left 

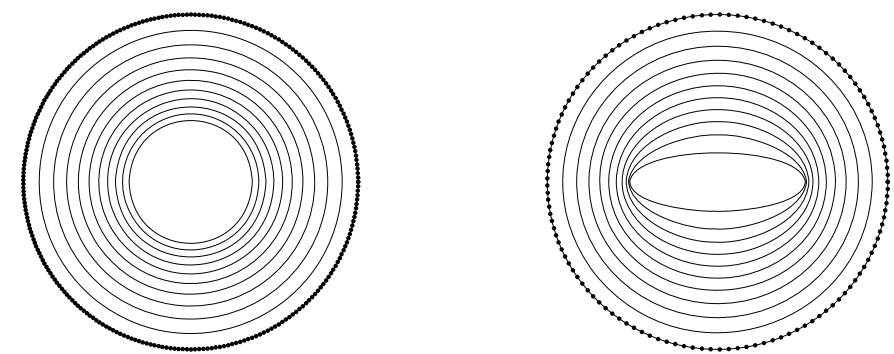

FIG. 8. $\vec{X}(t)$ for $t=0,0.1, \ldots, T=1$ for the inverse mean curvature flow of a circle (left) and an ellipse (right).
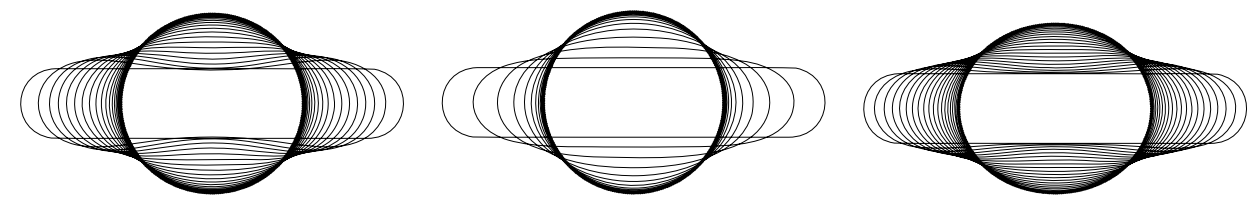

FIG. 9. Different evolutions for surface diffusion, area preserving mean curvature flow, and the intermediate flow (1.9), with $\alpha=\xi=1$. In each case, we plot $\vec{X}(t)$ for $t=0,1, \ldots, T=20$.

of Figure 8. The next experiment is for a $3: 1$ ellipse that expands to a circle. The discretization parameters are $N=128, \tau=10^{-3}$, and $T=1$; see the right-hand side of Figure 8.

3.1.2. Intermediate evolution laws. In this subsection, we report on numerical results for our approximation $(2.20 \mathrm{a}-\mathrm{c})$ of the intermediate evolution law (1.10). First, we compare the different evolutions of (1.2), (1.8) with $f(r):=r$, and (1.9) with $\alpha=\xi=1$, for an initial curve that is given by an elongated tube of dimensions $10 \times 1$. As discretization parameters for the schemes $(2.21 \mathrm{a}, \mathrm{b}),(2.4)$ with $(2.3 \mathrm{~b})$ and $f(r):=r$, and $(2.20 \mathrm{a}-\mathrm{c})$ we used $N=256, \tau=10^{-3}$, and $T=20$, and the corresponding results are shown in Figure 9. One can clearly see that while the curve that moves under area preserving mean curvature flow remains convex throughout the evolution, this is not the case for the other two evolution laws. The area losses for the respective schemes were $0.004 \%, 0.016 \%$, and $0.002 \%$. As noted before for $\alpha \rightarrow \infty$ and $\xi=1$, the solutions to (1.9) converge to solutions of (1.8), while $\xi \rightarrow \infty$ and $\alpha=1$ corresponds to the law (1.2). We now investigate this property numerically. To this end, we repeat the above experiments for $(2.20 \mathrm{a}-\mathrm{c})$ with $\xi=1, \alpha=1000$ and $\xi=1000, \alpha=1$, respectively. The results, for which the respective area losses were $0.008 \%$ and $0.004 \%$, can be seen in Figure 10. One can clearly see the similarity between these evolutions and their corresponding limits in Figure 9. We note also that the curve in the first evolution remains convex, while the curve for the second evolution does not.

3.2. Triple junctions. In the first experiment for triple junctions (see Figure 11) we simulate how two initially elliptic bubbles move under motion by mean curvature. Throughout, we assume equal surface energies, $\sigma_{i}=1, i=1 \rightarrow K_{C}$, unless stated otherwise. The plot on the left-hand side of Figure 11 shows the evolution 

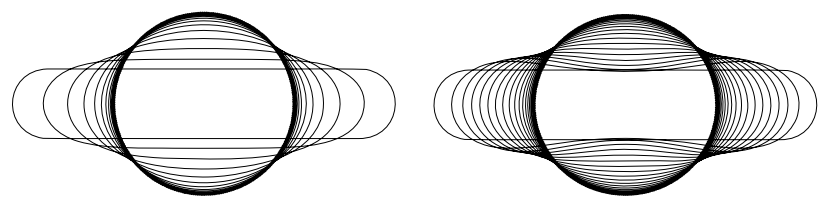

Fig. 10. The flow (1.9) with $\xi=1, \alpha=1000$ (left) and $\xi=1000, \alpha=1$ (right). In each case, we plot $\vec{X}(t)$ for $t=0,1, \ldots, T=20$.
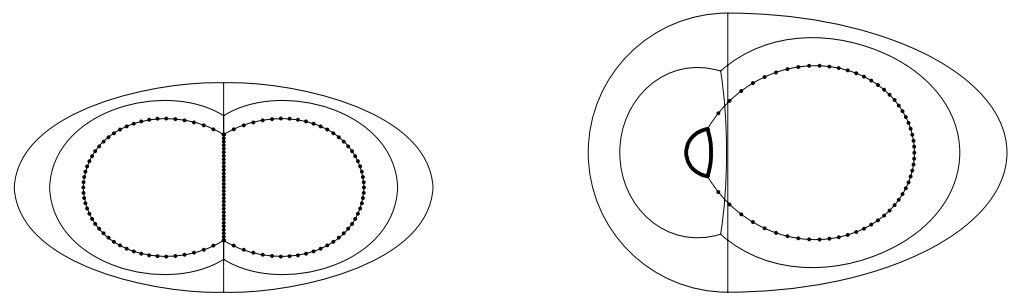

FIG. 11. $\vec{X}(t)$ for $t=0,0.2, T=0.4$. An equal area double bubble under mean curvature flow is shown on the left, and a nonequal area case on the right.
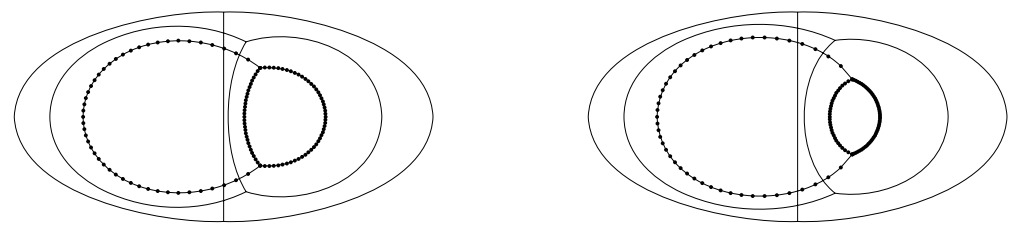

FIG. 12. $\vec{X}(t)$ for $t=0,0.2, T=0.4$. An equal area double bubble under mean curvature flow is shown for surface energies $\left(1,1, \frac{3}{2}\right)$ and $\left(1,1, \frac{7}{4}\right)$, respectively.

for two equal area bubbles, while the nonequal area case is displayed on the righthand side. The chosen parameters were $N=128, \tau=0.01$, and $T=0.4$. The initial shapes are given by two segments of a $2: 1$ ellipse with unit semiminor axis and a straight line, or a semicircle together with an elliptic segment from the above ellipse and a straight line, respectively. We repeated the experiment on the left-hand side of Figure 11 for different surface energies. The surface energies were chosen to be $\left(\sigma_{1}, \sigma_{2}, \sigma_{3}\right)=\left(1,1, \frac{3}{2}\right)$ and $\left(1,1, \frac{7}{4}\right)$. That means that the length of the curve $\Gamma_{3}$ is weighted more in the overall energy $|\Gamma|$, so that it will shorten faster during the evolution; see Figure 12.

The next experiment is for motion by surface diffusion. The initial setup, as depicted in Figure 13, consists of a segmentation of the $[-1,1]^{2}$ square, with the middle segments having width 0.3 . Our discretization parameters were $N=256$, $\tau=10^{-3}$, and $T=0.04$. The evolution leads to one curve segment shrinking to a single point. Of course, our approximation cannot compute beyond that singularity. However, on the right-hand side of Figure 13 we show how, after a topological change, the evolution continues to a numerically steady state. This numerical result was 

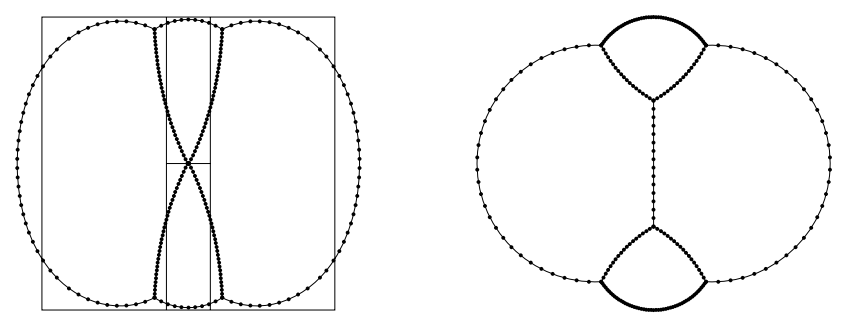

FIG. 13. A curve shrinks to a point: $\vec{X}(t)$ for $t=0, T$ (left). The eventual numerically steady state after a change of topology (right).
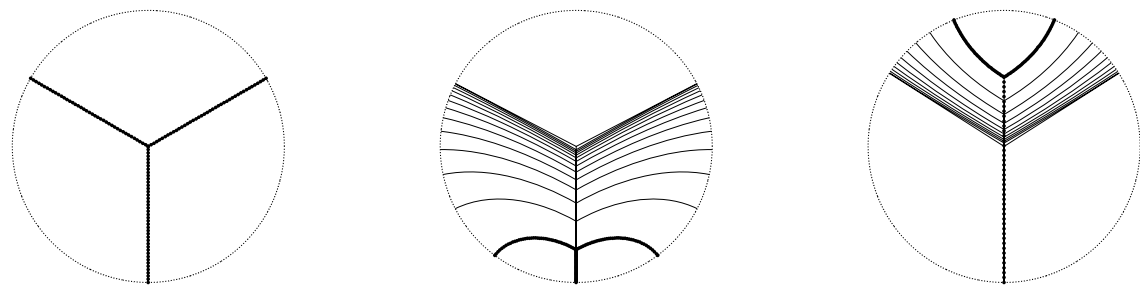

FIG. 14. A triple junction inside the unit circle moving under mean curvature flow.

obtained by restarting the computation from a curve network that was topologically equivalent to the steady state, and with enclosed regions having the same area as before, i.e., $0.7,0.3,0.7$, and 0.3 .

3.3. Boundary intersections. In the first experiment for curve networks with triple junctions and intersections with the external boundary (see Figure 14), we simulate how an initial "letter Y" inside the unit circle moves under motion by mean curvature. We varied the initial angle $\theta_{3}$ between the two upper curves, which meet at the origin. Here and throughout, $\theta:=\left(\theta_{1}, \theta_{2}, \theta_{3}\right)$ is defined as the angles formed by the three curve segments meeting at a triple junction, and $\theta_{i}, i=1 \rightarrow 3$, denotes the angle opposite the curve $\Gamma_{i}$ at a triple junction; recall (1.15).

The values used for $\theta_{3}$ are $120^{\circ}, 125^{\circ}$, and $115^{\circ}$ with $\theta_{1}=\theta_{2}$. The first setup is a steady state for this law of motion, while the other two experiments show that the symmetric boundary intersections will either move up or down the unit circle. The chosen discretization parameters for $(2.33 \mathrm{a}, \mathrm{b})$ were $N=128, \tau=0.01$, and $T=1,2.6$, and 2 , respectively. The times shown in Figure 14 are $t=0,0.2, \ldots, T$. The maximum distance $d_{\partial \Omega}:=\max _{i=1 \rightarrow 3} \operatorname{dist}\left(\vec{X}_{i}^{M}(1), \partial \Omega\right)$ of the curve endpoints at time $T$ was $d_{\partial \Omega}=0,8.6 \times 10^{-3}$, and $1.8 \times 10^{-3}$, respectively. Moreover, note that eventually the solution for the latter two experiments becomes either a single point (on the boundary $\partial \Omega$ ) or a single straight line.

We repeated the last experiment inside the square $[-1,1]^{2}$. Note that in this case $F \notin C^{1}\left(\mathbb{R}^{2}\right)$, but as the points of the derivative discontinuities are never reached by the evolving curves, this has no effect on the evolution; this will always be true in all examples below for such $F$. Here no steady state solution exists for this law of motion. For the results see Figure 15 , where we chose as angles $\theta_{3}=180^{\circ}, 95^{\circ}, 85^{\circ}$ and integrated until time $T=1.4,2$, and 0.4 , respectively. We observe that in the first two cases the solution exhibits a traveling wave character. In fact, an exact solution can be found for this setup; see [27, p. 313]. Let $\gamma(\rho, t):=\frac{6}{\pi} \ln \left(\cos \left(\frac{\pi}{6}(1-\rho)\right)\right)-\frac{\pi}{6} t+1$ 

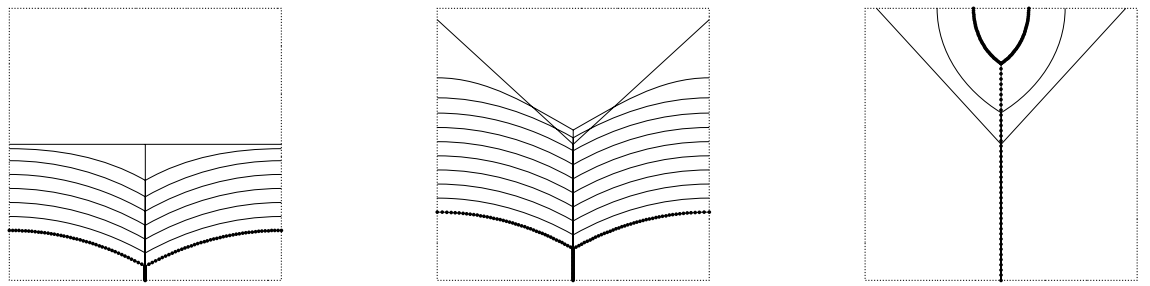

FIG. 15. A triple junction inside the square $[-1,1]^{2}$ moving under mean curvature flow.

TABLE 3

Absolute errors $\|\vec{X}-\vec{x}\|_{L^{\infty}}$ and triple junction angles for the test problem. On the right is shown an approximation of the exact solution (3.2) for $t=0,0.2, \ldots, 3$

\begin{tabular}{|r|c|c|}
\hline$N$ & $\|\vec{X}-\vec{x}\|_{L^{\infty}}$ & Angles $\theta=\left(\theta_{1}, \theta_{2}, \theta_{3}\right)$ \\
\hline 16 & $3.1156 \mathrm{e}-02$ & $(115.9,115.9,128.1)$ \\
32 & $8.7298 \mathrm{e}-03$ & $(118.2,118.2,123.7)$ \\
64 & $2.2632 \mathrm{e}-03$ & $(119.2,119.2,121.6)$ \\
128 & $5.7263 \mathrm{e}-04$ & $(119.6,119.6,120.7)$ \\
256 & $1.4362 \mathrm{e}-04$ & $(119.8,119.8,120.4)$ \\
512 & $3.5954 \mathrm{e}-05$ & $(119.9,119.9,120.2)$ \\
\hline
\end{tabular}

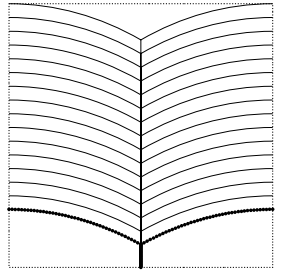

and

$\vec{x}_{1}(\rho, t)=\left(\begin{array}{c}-\rho \\ \gamma(\rho, t)\end{array}\right), \quad \vec{x}_{2}(\rho, t)=\left(\begin{array}{c}\rho \\ \gamma(\rho, t)\end{array}\right), \quad \vec{x}_{3}(\rho, t)=(1-\rho)\left(\begin{array}{c}0 \\ \gamma(0, t)\end{array}\right)+\rho\left(\begin{array}{c}0 \\ -1\end{array}\right)$.

Then $\vec{x}:=\left(\vec{x}_{1}, \vec{x}_{2}, \vec{x}_{3}\right)$ is a solution to $(1.12)$, (1.14a,b), and $(1.17 \mathrm{a}, \mathrm{b})$. We used this exact solution to perform a convergence test for our approximation $(2.33 \mathrm{a}, \mathrm{b})$. See Table 3, where we show the error $\|\vec{X}-\vec{x}\|_{L^{\infty}}$ on the time interval $[0, T]$ for $T=3$ and $\tau=0.5 h^{2}$. Here $\|\vec{X}-\vec{x}\|_{L^{\infty}}:=\max _{m=1 \rightarrow M}\left\|\vec{X}\left(t_{m}\right)-\vec{x}\left(\cdot, t_{m}\right)\right\|_{L^{\infty}}$ with $\left\|\vec{X}\left(t_{m}\right)-\vec{x}\left(\cdot, t_{m}\right)\right\|_{L^{\infty}}:=\max _{i=1 \rightarrow 3} \max _{j=0 \rightarrow N_{i}} \min _{\rho \in I}\left|\vec{X}_{i}^{m}\left(q_{j}^{i}\right)-\vec{x}_{i}\left(\rho, t_{m}\right)\right|$ is computed by employing a Newton method for the curved segments of the true solution. The evolution for $N=128$ is shown on the right of Table 3 .

3.3.1. Surface diffusion with boundary intersections. We repeated the above type of experiments for motion by surface diffusion, i.e., the approximation $(2.50 \mathrm{a}, \mathrm{b})$ of the system $(1.19)-(1.22)$ and $(1.17 \mathrm{a}, \mathrm{b})$. In the first experiment we simulate how an initial "letter $\mathrm{Y}$ " inside the unit circle moves under motion by surface diffusion. We varied the initial angle $\theta_{3}$ between the two upper curves at the origin with values $120^{\circ}, 180^{\circ}$, and $45^{\circ}$. While the first setup is already a steady state for this law of motion, the other two experiments soon reach a steady state. The chosen parameters were $N=128, \tau=10^{-4}$, and $T=0.05$. In Figure 16 we show $\vec{X}(t)$ at times $t=0, T$. Note that the maximum distance of the curve endpoints to the external boundary $\partial \Omega$ at time $T$ was $d_{\partial \Omega}=0,3 \times 10^{-5}$, and $8 \times 10^{-5}$, respectively. The results for the same experiments inside the domain $\Omega=[-1,1]^{2}$ can be found in Figure 17 . We note that here $d_{\partial \Omega}=0$ in each case, as the external boundary consists of straight lines.

We repeated the experiment on the left-hand side of Figure 16 for different surface energies. The surface energies were chosen to be $\left(\sigma_{1}, \sigma_{2}, \sigma_{3}\right)=\left(1,1, \frac{3}{2}\right)$ and $\left(1,1, \frac{7}{4}\right)$, respectively. We observe that as the length of the curve $\Gamma_{3}$ is weighted 

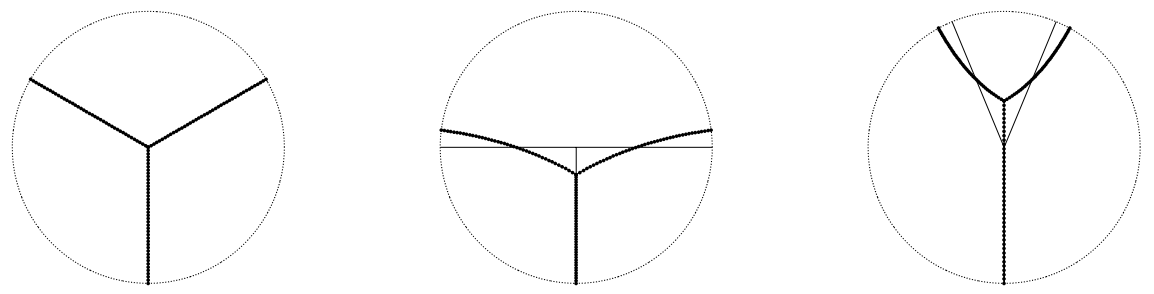

FIG. 16. A triple junction inside the unit circle moving under motion by surface diffusion.
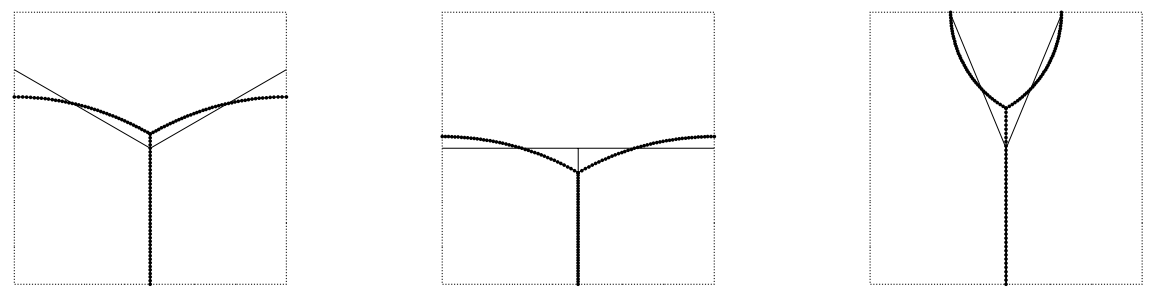

FIG. 17. A triple junction inside the square $[-1,1]^{2}$ moving under motion by surface diffusion.
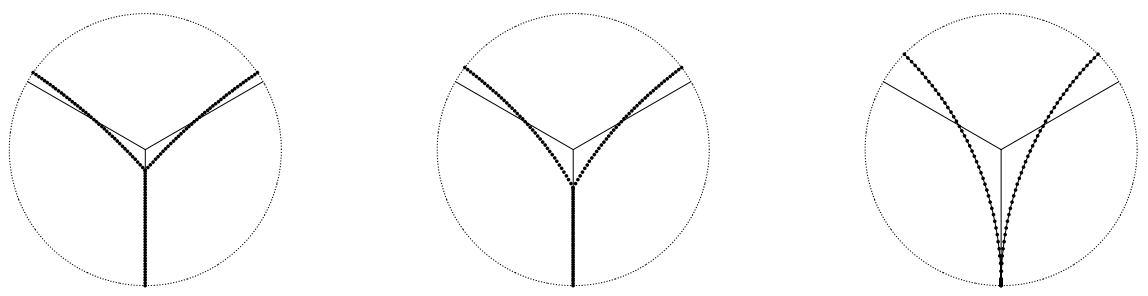

FIG. 18. A triple junction inside the unit circle moving under motion by surface diffusion for surface energies $\left(1,1, \frac{3}{2}\right),\left(1,1, \frac{7}{4}\right)$, and $(1,1,2)$.

more in the overall energy $|\Gamma|$, it shortens in comparison to the other two curves during the evolution; see Figure 18. The observed angles at the triple junction are $\theta=(138.4,138.4,83.2)$ and $\theta=(150.6,150.6,58.8)$. (Note that Young's law yields $\theta=(138.6,138.6,82.8)$ and $\theta=(151,151,57.9)$, respectively, for the exact solution.) Finally, we give the same evolution for the surface energies $(1,1,2)$ on the right-hand side of Figure 18. Here in the true steady state the curve $\Gamma_{3}$ has shrunk to a point on the boundary $\partial \Omega$, which, as it represents a change of topology, our approximation cannot compute. We give a plot of $\vec{X}(T)$ for $T=0.5$, when the observed triple junction angles are $\theta=(178.2,178.2,3.7)$.

An example with only two enclosed areas can be seen in Figure 19. Here the initial curve is given by a straight line through the origin that forms an angle of $10^{\circ}$ with the $x$-axis inside a $2: 1$ elliptic domain $\Omega$ with unit semiminor axis. The chosen parameters were $N=128, \tau=10^{-4}, T=2$. On the left-hand side of Figure 19 we plot $\vec{X}(t)$ at times $t=0,0.05, \ldots, T=2$. The right-hand side shows a similar experiment, where the initial curve goes through the point $\left(0,-\frac{1}{2}\right)^{T}$. The parameters were $N=32$, $\tau=10^{-3}$, and $T=10$, and we display $\vec{X}(t)$ at times $t=0,0.5, \ldots, T=10$. In both cases the evolution finds the minimum of a corresponding partitioning problem in which the length of the interface between two phases is minimized subject to an area constraint for the phases. 


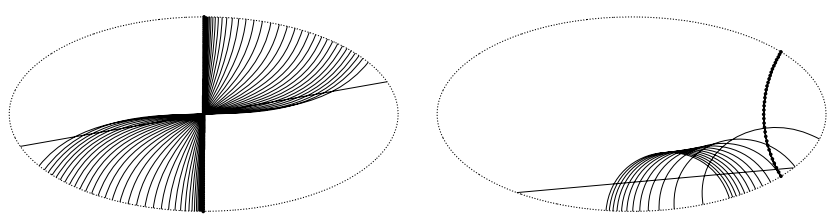

FIG. 19. A curve inside an elliptic domain moving under motion by surface diffusion.
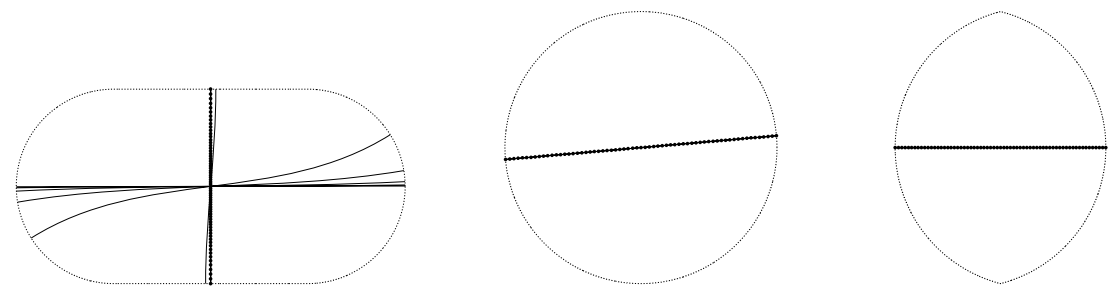

FIG. 20. Initially straight lines that are unstable (left), neutral (middle), or stable (right). On the left, $\vec{X}(t)$ at times $t=0,2, \ldots, 16$. The remaining plots show $\vec{X}(t)$ at times $t=0,6$.

Next, we investigate numerically the stability of some of the setups discussed in [25]. We start by considering a domain $\Omega$ that has a boundary with piecewise constant curvature. The analysis in [25, Figure 6$]$ predicts that a straight line inside $\Omega$ is only stable if it is sufficiently short. In the situation considered in Figure 20, it must not exceed twice the radius of the two arcs. In the first experiment, the total dimensions of the domain are $4 \times 2$. The initial line has a slope of $0.1^{\circ}$, and as the straight line on the $x_{1}$-axis is not stable, it evolves to a straight line on the $x_{2}$-axis. If, however, the dimensions of the domain are only $\frac{3}{2} \times 2$, then the straight line is stable, and this is seen in the corresponding experiment. The case where the domain is a circle is neutral, as any straight line through the origin is a steady state solution. As an example, we show an initially straight line with slope $5^{\circ}$, which does not change under the surface diffusion flow. The final solutions depicted in Figure 20 are all numerically steady states.

The next experiment investigates the behavior for the setup in [25, Figure 7]. In each case, we use a domain where the lower boundary follows a sine shape. In particular, the lower boundary is given by $x_{2}=-1+\frac{1}{8} \sin \left(2 \pi x_{1}\right)$. The initial profile is always a semicircle with radius 0.5. Depending on the sign of the curvature of the domain boundary, that solution is either stable or unstable. We investigate the stability numerically, by using $\vec{z}=\left(\mp 0.24,-1 \pm \frac{1}{8}\right)$ as the center of the half-circle. Note that $\vec{z}=\left(\mp \frac{1}{4},-1 \pm \frac{1}{8}\right)$ corresponds to a steady state. The results are shown in Figure 21, where we also include the neutral case of a semicircle attached to a straight part of the boundary.

Next, we performed computations with three enclosed areas and two vertices on the external boundary $\partial \Omega$; see Figure 22 . Starting with an initially circular area (with radius 0.75 ) that is connected by two straight lines to the external boundary inside a $2: 1$ rectangular domain, we observe different steady state solutions depending on the chosen surface energies $\sigma=\left(\sigma_{1}, \sigma_{2}, \sigma_{3}, \sigma_{4}\right)$. We note that for a physically relevant setup $\sigma_{1}=\sigma_{4}$, where $\Gamma_{1}$ and $\Gamma_{4}$ are the straight line curve segments. We used the 

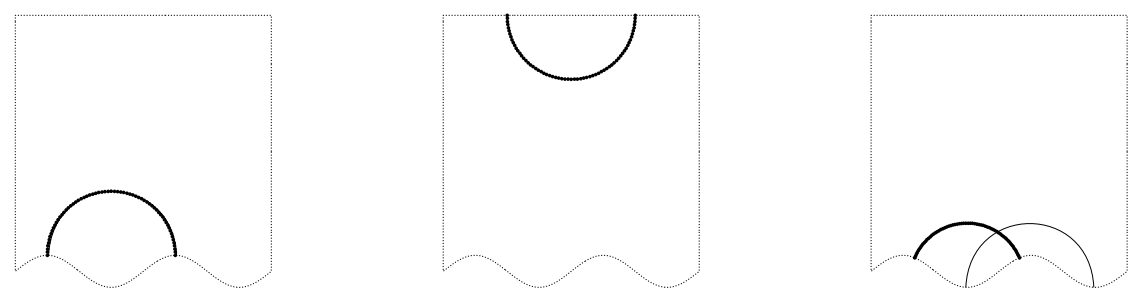

FIG. 21. Stable (left) and unstable (right) setup. In the middle a neutral situation is shown. Each plot shows $\vec{X}(t)$ at times $t=0,1$.
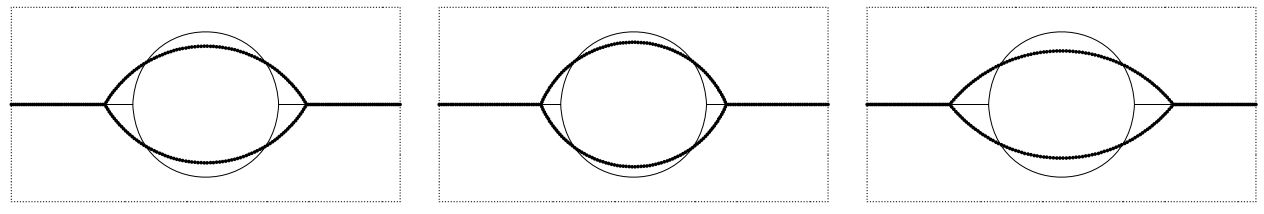

FIG. 22. Steady state solutions for different angles $\theta_{1}$.
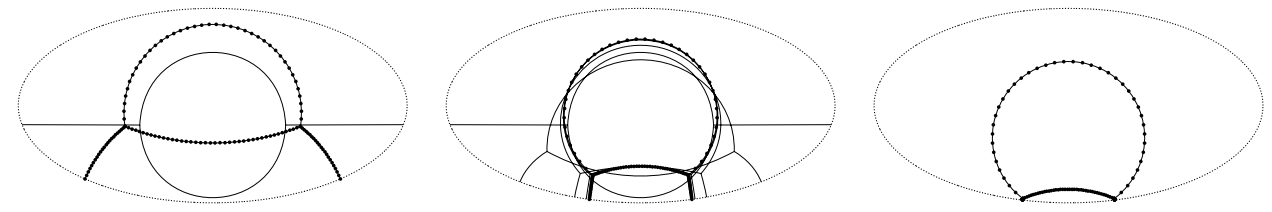

FIG. 23. Solution for circular setup for pure surface diffusion (left) and combined mean curvature and surface diffusion inside an elliptic domain. In the middle $\vec{X}^{m}(t)$ at times $t=0,0.5, \ldots, 2$ and on the right $\vec{X}^{m}(T)$ at $T=40$.

values $\sigma=(1, \lambda, \lambda, 1)$ with $\lambda=1, \frac{\sqrt{7}}{2},\left(\frac{5}{8}\right)^{\frac{1}{2}}$, corresponding to [7, Figures 3 and 8], so that the true triple junction angle is $\theta_{1}=120^{\circ}, 135^{\circ}, 102^{\circ}$, respectively. The chosen discretization parameters for each experiment were $N=256, \tau=10^{-4}$, and $T=0.2$. The observed angles inside the circular area were $\theta_{1}=118.6^{\circ}, 133.9^{\circ}$, and $100.2^{\circ}$, respectively.

For later purposes, we repeated the first experiment in Figure 22, but now inside a 2:1 elliptic domain and with the initial profile at the height $y=-0.2$. The chosen parameters were $N=128, \tau=10^{-3}$, and $T=2$, by which time the numerical solution has reached a steady state. The results are shown on the left-hand side of Figure 23.

Next, we report on some experiments for (2.50a) and (2.34), i.e., for the surface diffusion of a curve network with specified contact angles $\alpha_{i}$ that the curves form with the external boundary $\partial \Omega$. Here we use as initial data a "letter $Y$ " inside the unit circle, similarly to Figure 14 . We varied the angle $\alpha_{i}, i=1 \rightarrow 3$, that the three curves form with the external boundary. In Figure 24 we show the solution $\vec{X}(t)$ at times $t=0, T$ for the values $\alpha_{1}=\alpha_{2}=\alpha_{3}=\alpha$ with $\alpha=60^{\circ}, 30^{\circ}$, and $5^{\circ}$. The chosen discretization parameters were $N=128, \tau=10^{-3}$, and $T=0.1$. The same experiments inside the square $[-1,1]^{2}$, where the initial profile is now a "letter $\mathrm{T}$, 

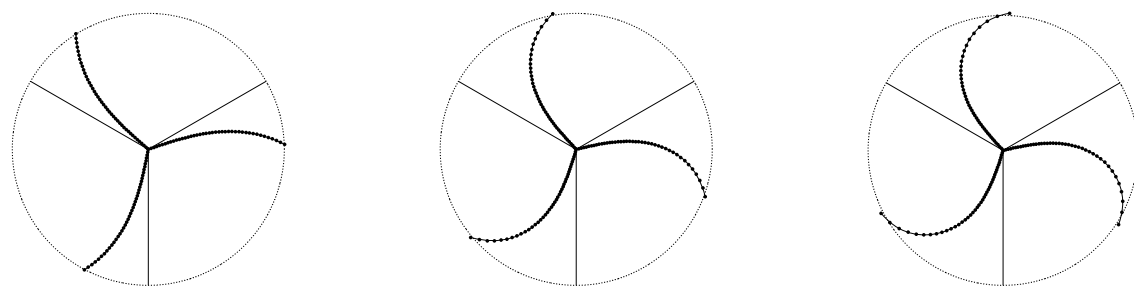

FIG. 24. A triple junction inside the unit circle with different contact angles at $\partial \Omega$.
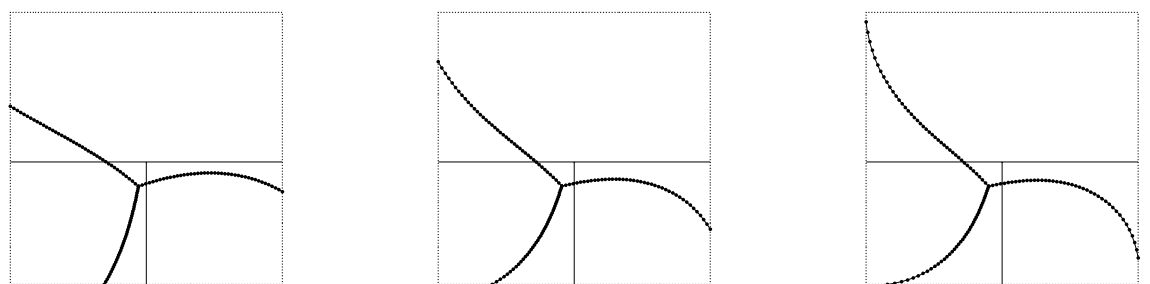

FIG. 25. A triple junction inside the square $[-1,1]^{2}$ with different contact angles at $\partial \Omega$.
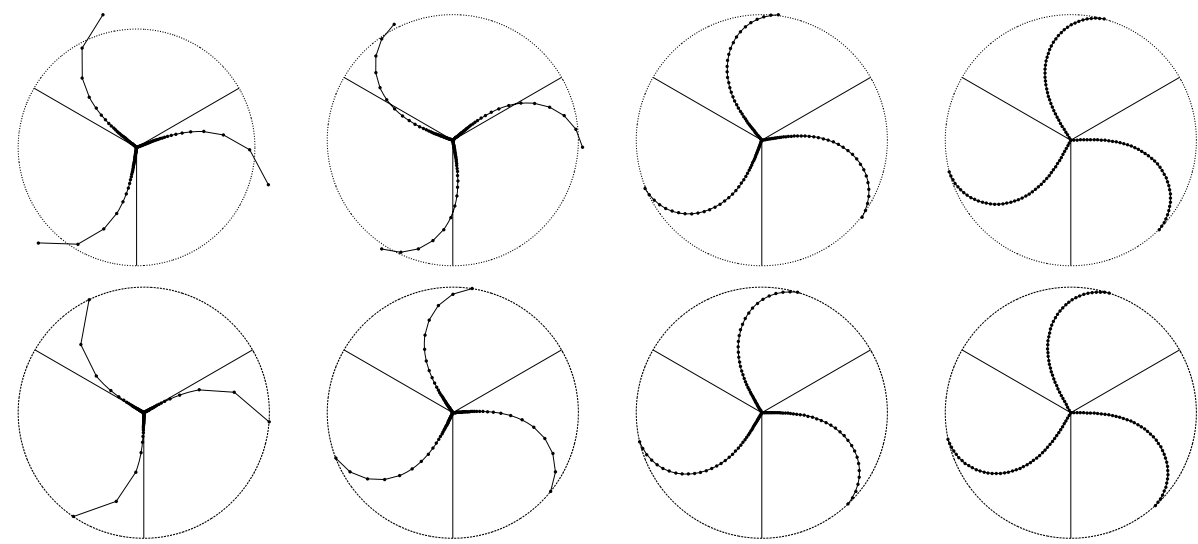

FIG. 26. Tangential movement and boundary vertex mislocation for different time step sizes, $\tau=10^{-k}, k=2 \rightarrow 5$. Results for $(2.50 \mathrm{a}, \mathrm{b})$ (top) and the corresponding scheme with a projection onto $\partial \Omega$ at each time step (bottom).

can be seen in Figure 25.

We note that in practice we observe a tangential movement of mesh points away from the boundary intersection towards the triple junction. On the other hand, we have mentioned in Remark 2.5 that it was shown in [6] that for a semidiscrete in time approximation the vertices equidistribute along each curve $\Gamma_{i}^{h}, i=1 \rightarrow 3$. Hence for $\tau$ sufficiently small, we expect the parameterizations $\vec{X}_{i}^{m}$ to become more and more uniform. We investigate this behavior with the following set of experiments. We repeat the last experiment in Figure 24 and integrate until time $T=1$ for different choices of $\tau=10^{-k}, k=2 \rightarrow 5$. In the top row of Figure 26 we see clearly that the distribution of vertices improves as $\tau$ decreases. For comparison, we include the results for the same set of experiments for a version of our scheme, where one employs 

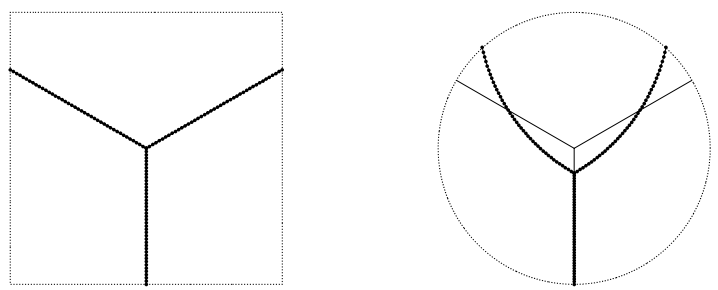

FIG. 27. A steady state solution for given boundary contact angles inside the square $[-1,1]^{2}$, and the corresponding evolution inside the unit circle.

a projection step that orthogonally projects the boundary vertices of $\vec{X}^{m+1}$ onto $\partial \Omega$ at every time step. As can be seen from the results in the bottom row of Figure 26, for small time step sizes the results are virtually indistinguishable, while for large time steps it can be beneficial to employ the described projection step.

Finally, we provide an experiment for a steady state inside the domain $\Omega=$ $[-1,1]^{2}$. The chosen contact angles are $\alpha_{1}=60^{\circ}, \alpha_{2}=120^{\circ}$, and $\alpha_{3}=90^{\circ}$, so that

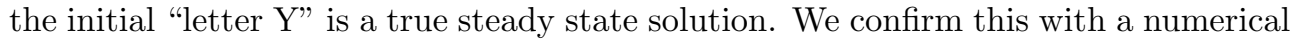
experiment for (2.50a) and (2.34) with $N=128, \tau=10^{-3}$, and $T=0.5$; see Figure 27 , where we complement this result with the corresponding evolution inside the unit circle.

3.3.2. Combined surface diffusion and mean curvature flow. In this subsection, we report on numerical results for the approximation (2.53a,b) of (1.23). First, we repeated the experiment reported on earlier, shown on the left-hand side of Figure 23, but now for a combined motion of surface diffusion and mean curvature flow. In particular, we prescribe surface diffusion on the two initially circular curves, while the initially straight-lined curves experience motion by mean curvature. See Figure 23 for the results, where we plot $\vec{X}^{m}(t)$ at different times. The chosen parameters were $N=128, \tau=10^{-3}$, and $T=40$. One can clearly see the very different evolution compared to the pure surface diffusion flow, and note in particular that each of the two curves moving under motion by mean curvature now shrink to a single point (on the boundary $\partial \Omega$ ).

The next experiment is motivated by the considerations on a traveling wave solution to (1.23) that was first mentioned in [36] and which plays a major role in the study of grain boundary motion; see also [33] and [7, Figure 7]. The computations shown in Figure 28 start with three curves meeting at a single triple junction, of which the two horizontal ones experience motion by surface diffusion, while the third curve undergoes motion by mean curvature. As surface energies we chose $\sigma=\left(1,1, \sigma_{3}\right)$ with $\sigma_{3}=1$ and $\sigma_{3}=\frac{3}{2}$, respectively. Moreover, the evolution law for the curve moving under motion by mean curvature is here given by $\mathcal{V}_{3}=\mu_{3} \sigma_{3} \varkappa_{3}$ in place of (1.12), where for the mobility we chose either $\mu_{3}=1$ or $\mu_{3}=2$. Note that our approximation $(2.53 \mathrm{a}, \mathrm{b})$ can easily be adapted to this situation by replacing $\sigma_{3}$ with $\mu_{3} \sigma_{3}$ in (2.53a). The domain $\Omega$ is an $8 \times 2$ rectangle. The chosen discretization parameters were $N=256$ and $\tau=10^{-3}$.

3.4. Quadruple junctions. Here we consider a setup of three enclosed areas meeting at two triple junctions and one quadruple junction. A physical interpretation of this configuration is an alloy material with two grains and two phases, similar to the situation considered in [10, 26]. For the initial curves depicted in Figure 29 this 

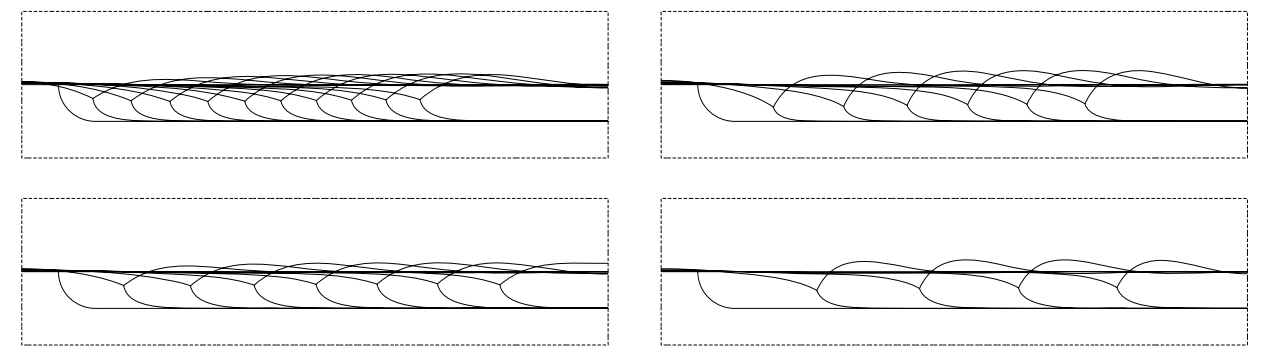

FIG. 28. Traveling wave solutions $\vec{X}^{m}(t)$ at times $t=0,0.1, \ldots, T$, with $T=1,0.6,0.7$, and 0.4 , respectively. Surface energies are $\sigma_{3}=1$ (left) and $\sigma_{3}=\frac{3}{2}$ (right), with mobilities $\mu_{3}=1$ (top) and $\mu_{3}=2$ (bottom).
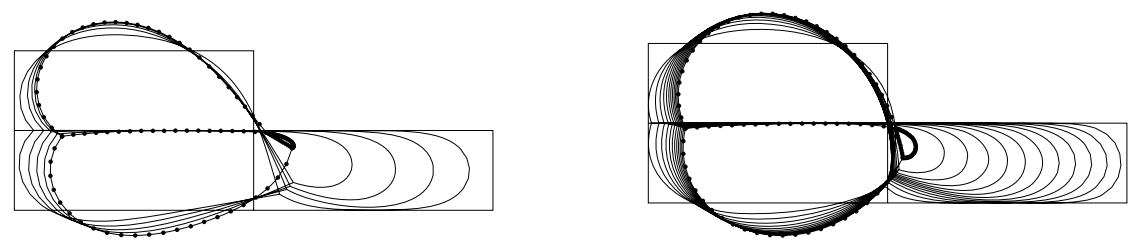

FIG. 29. $\vec{X}(t)$ for $t=0,0.1, \ldots, T$ for a quadruple junction with $\lambda=\frac{3}{2}$ (left) and $\lambda=\frac{2}{3}$ (right).
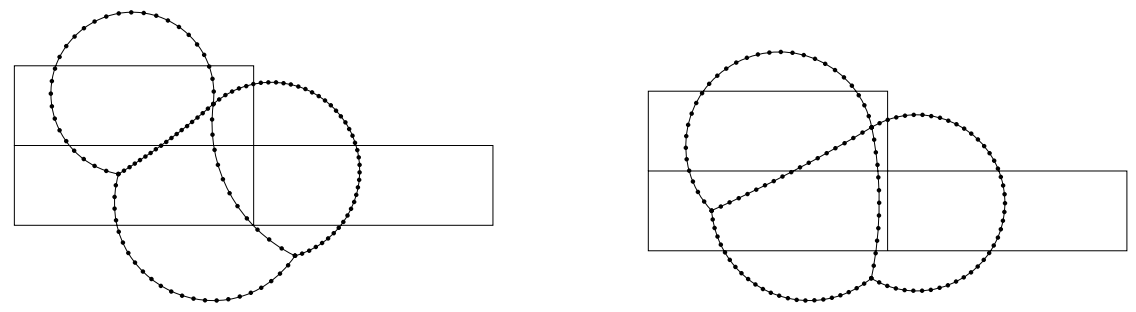

FIG. 30. $\vec{X}(t)$ for $t=0, T$ for a quadruple junction with $\lambda=\frac{3}{2}$ (left) and $\lambda=\frac{2}{3}$ (right).

means that the two left enclosed areas represent phase $B$, while the rest of the plane is made up of phase $A$. Moreover, the lower two enclosed areas represent grain I, with the rest of the domain being grain II. Then we have motion by surface diffusion for the interfaces $\Gamma_{1}$ and $\Gamma_{3}$ separating the two phases, and mean curvature flow for the interfaces $\Gamma_{2}$ and $\Gamma_{4}$ between the two grains. The surface energies were chosen to be $\sigma_{S D}=1$ for the curves moving by surface diffusion and $\sigma_{M C}=\lambda$ for the remaining curves. Two evolutions for $\lambda=\frac{3}{2}$ and $\lambda=\frac{2}{3}$, where the initial curves create a collection of 3:1 rectangles, can be seen in Figure 29. The discretization parameters were $N=128, \tau=10^{-3}$, and $T=0.49$ and $T=1.2$, respectively. In both evolutions we can see that the two curves enclosing grain I in phase $A$ eventually shrink to a point. The change of topology needed to continue the evolution is beyond the scope of our direct parametric approximation. We also give the corresponding numerical steady states for the pure surface diffusion flow version of this experiment in Figure 30. The discretization parameters were $N=128, \tau=10^{-3}$, and $T=8$. 
Finally, we remark that, on recalling $\sigma_{S D}=1$ and $\sigma_{M C}=\lambda$, the balance of forces equation (1.16) becomes $\left(\vec{\tau}_{1}+\vec{\tau}_{3}\right)+\lambda\left(\vec{\tau}_{2}+\vec{\tau}_{4}\right)=\overrightarrow{0}$. It follows from this that $\theta_{1}=\theta_{3}$ and $\theta_{2}=\theta_{4}$, where $\theta_{i}:=\varangle\left(\vec{\tau}_{i}, \vec{\tau}_{i+1}\right), i=1 \rightarrow 3$, and $\theta_{4}:=\varangle\left(\vec{\tau}_{4}, \vec{\tau}_{1}\right)$, as adding the four vectors $\vec{\tau}_{1}, \lambda \vec{\tau}_{2}, \vec{\tau}_{3}, \lambda \vec{\tau}_{4}$ in the plane leads to a 4-polygon with two opposite sides having length 1 and two opposite sides having length $\lambda$. This implies that the 4polygon is a parallelogram, and hence opposite angles in the parallelogram have to be equal. This fact can be seen clearly in the numerical computations. For example, on labeling the curves anticlockwise starting from the top most one, the angles observed in Figure 30 at the quadruple junction are $\theta=(133.1,44.6,133.7,48.6)$ and $\theta=$ $(107.5,69.6,105.8,77.1)$, respectively; i.e., the stated angle condition is approximately satisfied.

4. Conclusions. We have presented a fully practical finite element approximation for the (combined) motion by mean curvature and motion by surface diffusion of curves in $\mathbb{R}^{2}$, as well as for other related second and fourth order geometric evolution equations. Our scheme can handle both triple and quadruple junction points between different curves, and intersections of curves with a fixed external boundary $\partial \Omega$. To our knowledge, this is the first such scheme in the literature. Moreover, the presented scheme intrinsically moves the vertices tangentially along the curves, so that no artificial redistribution of vertices is necessary in practice. Finally, we note that a generalization of the presented method to geometric flows of two-dimensional hypersurfaces in $\mathbb{R}^{3}$ will be considered in the forthcoming manuscript [5]. Moreover, extending our schemes for curves and hypersurfaces to include the case of fully anisotropic surface energies is the subject of our ongoing research in this area.

\section{REFERENCES}

[1] L. Alvarez, F. Guichard, P.-L. Lions, and J.-M. Morel, Axioms and fundamental equations of image processing, Arch. Ration. Mech. Anal., 123 (1993), pp. 199-257.

[2] S. Angenent, G. Sapiro, and A. Tannenbaum, On the affine heat equation for nonconvex curves, J. Amer. Math. Soc., 11 (1998), pp. 601-634.

[3] E. BÄnsch, P. Morin, And R. H. Nochetto, A finite element method for surface diffusion: The parametric case, J. Comput. Phys., 203 (2005), pp. 321-343.

[4] J. W. Barrett, H. Garcke, And R. Nürnberg, Finite element approximation of a phase field model for multicomponent surface diffusion, 2007, in preparation.

[5] J. W. Barrett, H. Garcke, and R. Nürnberg, On the Parametric Finite Element Approximation of Evolving Hypersurfaces in $\mathbb{R}^{3}$, Preprint No. 18/2006, University Regensburg, Regensburg, Germany, 2006.

[6] J. W. Barrett, H. Garcke, and R. Nürnberg, A parametric finite element method for fourth order geometric evolution equations, J. Comput. Phys., 222 (2007), pp. 441-467.

[7] J. W. Barrett, H. Garcke, And R. Nürnberg, A phase field model for electromigration of intergranular voids, Interfaces Free Bound., to appear.

[8] L. Bronsard AND F. ReITich, On three-phase boundary motion and the singular limit of a vector-valued Ginzburg-Landau equation, Arch. Ration. Mech. Anal., 124 (1993), pp. 355379.

[9] L. Bronsard and B. T. R. Wetton, A numerical method for tracking curve networks moving with curvature motion, J. Comput. Phys., 120 (1995), pp. 66-87.

[10] J. W. CAHN, Stability, microstructural evolution, grain growth, and coarsening in a twodimensional two-phase microstructure, Acta Metall., 39 (1991), pp. 2189-2199.

[11] J. W. Cahn, E. A. Holm, and D. J. Srolovitz, Modeling microstructural evolution in twodimensional two-phase microstructures, Mater. Sci. Forum, 94-96 (1992), pp. 141-158.

[12] J. W. CAHN AND A. NoviCK-CoHen, Evolution equations for phase separation and ordering in binary alloys, J. Statist. Phys., 76 (1994), pp. 877-909.

[13] K. Deckelnick And G. Dziuk, On the approximation of the curve shortening flow, in Calculus of Variations, Applications, and Computations (Pont-à-Mousson, 1994), Pitman Res. Notes Math. 326, Longman Scientific and Technical, Harlow, UK, 1995, pp. 100-108. 
[14] K. Deckelnick, G. Dziuk, and C. M. Elliott, Computation of geometric partial differential equations and mean curvature flow, Acta Numer., 14 (2005), pp. 139-232.

[15] K. Deckelnick And C. M. Elliott, Finite element error bounds for a curve shrinking with prescribed normal contact to a fixed boundary, IMA J. Numer. Anal., 18 (1998), pp. 635654 .

[16] G. DzIUK, An algorithm for evolutionary surfaces, Numer. Math., 58 (1991), pp. 603-611.

[17] G. DzIUK, Convergence of a semidiscrete scheme for the curve shortening flow, Math. Models Methods Appl. Sci., 4 (1994), pp. 589-606.

[18] G. DzIuk, Numerical schemes for the mean curvature flow of graphs, in Variations of Domain and Free-Boundary Problems in Solid Mechanics (Paris, 1997), Solid Mech. Appl. 66, Kluwer Academic Publishers, Dordrecht, The Netherlands, 1999, pp. 63-70.

[19] G. Dziuk, E. Kuwert, AND R. Schätzle, Evolution of elastic curves in $\mathbb{R}^{n}$ : Existence and computation, SIAM J. Math. Anal., 33 (2002), pp. 1228-1245.

[20] C. M. Elliott and H. Garcke, Existence results for diffusive surface motion laws, Adv. Math. Sci. Appl., 7 (1997), pp. 465-488.

[21] J. Escher, Y. GiGA, AND K. ITO, On a limiting motion and self-intersections of curves moved by the intermediate surface diffusion flow, Nonlinear Anal., 47 (2001), pp. 3717-3728.

[22] X. Feng and A. Prohl, Finite Element Approximations of the Inverse Mean Curvature Flow Arising from the General Relativity, FIM Preprint, ETH Zurich, Zurich, Switzerland, 2005.

[23] R. Finn, Equilibrium Capillary Surfaces, Grundlehren Math. Wiss. 284, Springer, New York, 1986.

[24] P. Freudenberger, Tripel- und Quadrupelpunkte im Krümmungsfluß, Master's thesis, Mathematics Department, University of Bonn, Bonn, Germany, 1997.

[25] H. Garcke, K. Ito, AND Y. KohSAKa, Linearized stability analysis of stationary solutions for surface diffusion with boundary conditions, SIAM J. Math. Anal., 36 (2005), pp. 10311056.

[26] H. Garcke, B. Nestler, And B. Stinner, A diffuse interface model for alloys with multiple components and phases, SIAM J. Appl. Math., 64 (2004), pp. 775-799.

[27] H. Garcke, B. Nestler, and B. Stoth, A multiphase field concept: Numerical simulations of moving phase boundaries and multiple junctions, SIAM J. Appl. Math., 60 (1999), pp. 295-315.

[28] H. Garcke And A. Novick-Cohen, A singular limit for a system of degenerate Cahn-Hilliard equations, Adv. Differential Equations, 5 (2000), pp. 401-434.

[29] R. Geroch, Energy extraction, Ann. New York Acad. Sci., 224 (1973), pp. 108-117.

[30] M. R. Hestenes, Pseudoinverses and conjugate gradients, Comm. ACM, 18 (1975), pp. 40-43.

[31] G. Huisken and T. Ilmanen, The inverse mean curvature flow and the Riemannian Penrose inequality, J. Differential Geom., 59 (2001), pp. 353-437.

[32] P. S. JANG, On the positive energy conjecture, J. Math. Phys., 17 (1976), pp. 141-145

[33] J. Kanel, A. Novick-Cohen, AND A. Vilenkin, Coupled surface and grain boundary motion: Nonclassical traveling wave solutions, Adv. Differential Equations, 9 (2004), pp. 299-327.

[34] B. Merriman, J. K. Bence, and S. J. Osher, Motion of multiple functions: A level set approach, J. Comput. Phys., 112 (1994), pp. 334-363.

[35] K. Mikula AND D. ŠEvČOviČ, Evolution of plane curves driven by a nonlinear function of curvature and anisotropy, SIAM J. Appl. Math., 61 (2001), pp. 1473-1501.

[36] W. W. Muluins, The effect of thermal grooving on grain boundary motion, Acta Metall., 6 (1958), pp. 414-427.

[37] R. Neubauer, Ein Finiteelementeansatz für Krümmungsfluß von unter Tripelpunktbedingungen verbundenen Kurven, Master's thesis, Mathematics Department, University of Bonn, Bonn, Germany, 2002.

[38] E. PASCH, Numerische Verfahren zur Berrechnung von Krümmungsflüssen, Ph.D. thesis, Mathematics Department, University of Tübingen, Tübingen, Germany, 1998.

[39] J. Rubinstein, P. Sternberg, And J. B. Keller, Fast reaction, slow diffusion, and curve shortening, SIAM J. Appl. Math., 49 (1989), pp. 116-133.

[40] G. Sapiro and A. Tannenbaum, On affine plane curve evolution, J. Funct. Anal., 119 (1994), pp. $79-120$.

[41] K. A. Smith, F. J. Solis, And D. L. Chopp, A projection method for motion of triple junctions by levels sets, Interfaces Free Bound., 4 (2002), pp. 263-276.

[42] J. E. TAYLOR AND J. W. CAHN, Linking anisotropic sharp and diffuse surface motion laws via gradient flows, J. Statist. Phys., 77 (1994), pp. 183-197.

[43] B. Thaddey, Numerik für die Evolution von Kurven mit Tripelpunkt, Master's thesis, Mathematics Department, University of Freiburg, Freiburg, Germany, 1999.

[44] H.-K. Zhao, B. Merriman, S. Osher, and L. Wang, Capturing the behavior of bubbles and drops using the variational level set approach, J. Comput. Phys., 143 (1998), pp. 495-518. 